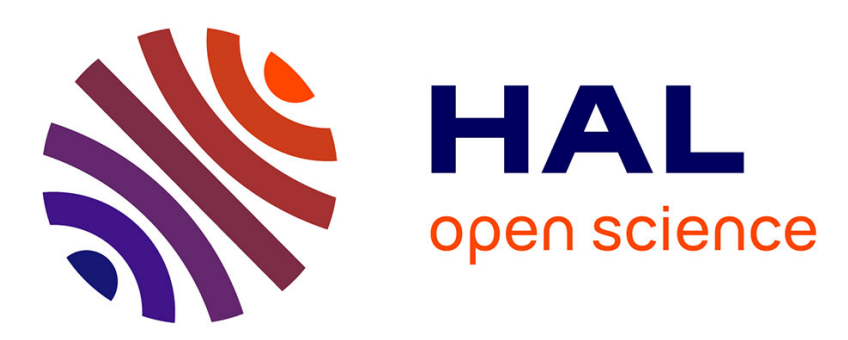

\title{
Non-Linear Simulations of Neoclassical Tearing Mode Control by Externally Driven RF Current and Heating, with application to ITER
}

Fabien Widmer, Patrick Maget, Olivier Février, Hinrich Lütjens, Xavier Garbet

\section{To cite this version:}

Fabien Widmer, Patrick Maget, Olivier Février, Hinrich Lütjens, Xavier Garbet. Non-Linear Simulations of Neoclassical Tearing Mode Control by Externally Driven RF Current and Heating, with application to ITER. Nuclear Fusion, 2019, 59 (10), pp.106012. 10.1088/1741-4326/ab300f . cea02092840

\section{HAL Id: cea-02092840 https://hal-cea.archives-ouvertes.fr/cea-02092840}

Submitted on 8 Apr 2019

HAL is a multi-disciplinary open access archive for the deposit and dissemination of scientific research documents, whether they are published or not. The documents may come from teaching and research institutions in France or abroad, or from public or private research centers.
L'archive ouverte pluridisciplinaire HAL, est destinée au dépôt et à la diffusion de documents scientifiques de niveau recherche, publiés ou non, émanant des établissements d'enseignement et de recherche français ou étrangers, des laboratoires publics ou privés. 


\title{
Non-Linear Simulations of Neoclassical Tearing Mode Control by Externally Driven RF Current and Heating, with application to ITER
}

\author{
Fabien Widmer, Patrick Maget, Olivier Février ${ }^{1}$, Hinrich \\ Lütjens $^{2}$, Xavier Garbet \\ CEA, IRFM, F-13108 Saint Paul-lez-Durance, France. \\ 1 Swiss Plasma Center, Ecole Polytechnique Fédérale de Lausanne, CH-1015 \\ Lausanne, Switzerland. \\ ${ }^{2}$ Centre de Physique Théorique, Ecole Polytechnique, CNRS, France. \\ E-mail: patrick.maget@cea.fr
}

\begin{abstract}
Neoclassical Tearing Modes (NTM) must be controlled or suppressed to prevent a degradation of the energy confinement in tokamak plasmas. This can be done applying RF-current via Electron Cyclotron Current Drive (ECCD) and -heating $(\mathrm{ECRH})$ at the rational surface where the instability appears. Both the current and heating generated by the RF waves are known to provide a stabilizing effect on the magnetic island. In the present work, we address the issue of Neoclassical Tearing Mode stabilization by Heating and Current Drive in an ITER-like configuration, using a stiff transport model. From a revised Generalized Rutherford Equation, we revisit the criterion on the RF current and power required to stabilize an NTM, showing that the level of plasma background heating (residual heat sources) in ITER significantly lowers the benefit of the RF heating contribution. Nonlinear MagnetoHydroDynamic simulations with the XTOR code, where a stiff transport model as well as RF-power and -current drive are implemented, are performed to compute the NTM stabilization efficiency. The stabilization efficiency due to the RF current contribution is found to be less than theoretically predicted in the case of continuous application, but consistent with theory in the modulated control scheme, suggesting an enhanced destabilization at the X-point. The role of RF heating for continuous application is found to be moderate for the range of power envisaged in ITER, essentially because of the detrimental effect of residual heat sources. This numerical work confirms the capability of the ITER RF system to control the $(3,2)$ NTM, with a larger confidence for the modulated control scheme.
\end{abstract}




\section{Introduction}

The power produced by fusion devices is proportional to the square of the plasma beta $\beta$, the ratio of the plasma pressure over the magnetic pressure. A large $\beta$ is therefore desired in future machines such as ITER. An important task to ensure good performances of the device is the control of magneto-hydrodynamic (MHD) plasma instabilities, among which Neoclassical Tearing Modes (NTMs) deserve particular interest because of their broad range of impact, from energy confinement degradation to plasma disruption. These modes consist of magnetic island that are metastable, i.e. linearly stable and non linearly unstable above some critical island size. The linear stability essentially results from a large curvature effect associated with the large plasma $\beta$ [Kotschenreuther et al., 1985, Lütjens et al., 2001], while the nonlinear drive is provided by the self generated bootstrap current [Carrera et al., 1986].

As any magnetic island, NTMs can be controlled by a current source opposing the island current, and localized preferably at the O-point. This focused current source can be obtained either by directly driving an electron flow, or by heating the plasma, which in presence of a toroidal electric field results in an amplification of the ohmic current. Both mechanisms lead to a decay of the island size [Hegna and Callen, 1997]. In tokamaks, the use of RF waves at the Electron Cyclotron (EC) frequency has proved to be very efficient in this respect because of the narrow deposition width that can be obtained, as demonstrated in a large number of experiments [Maraschek, 2012], and verified in numerical simulations [Yu and Günter, 1998, Popov et al., 2002, Yu et al., 2000, Yu et al., 2004, Février et al., 2016, Février et al., 2017]. Although the best results are obtained when the $\mathrm{RF}$ beam is toroidally oriented to drive a current, the favorable effect of pure heating has also been demonstrated experimentally [Westerhof et al., 2007] and numerically [Kurita et al., 1994, Lazzari and Westerhof, 2009, Lazzari and Westerhof, 2010, Kim et al., 2016, Maget et al., 2018b].

The evaluation of the relative benefits of heating and current drive suggests that the current contribution is the most efficient on large tokamaks [Lazzari and Westerhof, 2009, Lazzari and Westerhof, 2010], and the heating contribution is generally neglected in island stabilization analyzes, either for present experiments or for ITER. However, the vanishing of turbulent transport inside a magnetic island was neglected until recently [Fitzpatrick, 2017], and numerous experiment observations report strong evidences of this phenomenon [Inagaki et al., 2004, Ida et al., 2012, Bardòczi et al., 2016, Bardòczi et al., 2017], also supported by numerical simulations of turbulence in presence of a magnetic island [Hornsby et al., 2010, Hornsby et al., 2011, Zarzoso et al., 2015, Hill et al., 2015, Izacard et al., 2016, Navarro et al., 2017, Agullo et al., 2017a, Agullo et al., 2017b]. The response of an island to a localized heating is indeed sensitive to the stiffness of the temperature profile due to turbulent transport properties. The island will respond strongly to limited power densities, up to the level where turbulence will be excited, and will not respond to further power density increase because a growing turbulence 
level will prevent further temperature increase. The implication is that the contribution of power density to the island stabilization can be much larger than anticipated with a non-stiff transport model when the RF power is moderate compared to the power flowing through the island position [Maget et al., 2018a]. But on the other hand, even a limited background heating, present before the RF system is used, can damp the control capability by localized heating [Maget et al., 2018b]. The RF heating contribution is therefore very much case dependent, and one of the goals of the present study is to evaluate it in an ITER-like configuration.

In the present work, we investigate issues related with the stabilization of the $(m=$ 3, $n=2$ ) NTM , with $m$ and $n$ the poloidal and toroidal mode numbers respectively, in an ITER-like configuration. In ITER, a dedicated system is designed for this purpose [Figini et al., 2015, Henderson et al., 2015, Poli et al., 2015, Poli et al., 2018], with a power of $13 \mathrm{MW}$ made available for the control of this island. We first address this question using a simple evolution equation for the magnetic island (Rutherford Equation), where the bootstrap drive and RF heat and current drive terms are considered (section 2). A criterion on the required RF current drive and power needed for full stabilization is derived and illustrated for the ITER situation. In a second part, we present full MHD non linear simulations using the XTOR code [Lütjens and Luciani, 2010] where a stiff transport model [Maget et al., 2018b] and RFheating and -current drive are implemented [Février et al., 2016, Février et al., 2018]. The MHD model that is considered includes ion and electron diamagnetic rotations as well as an ad-hoc bootstrap current, and we consider a simplified ITER-like configuration (section 3) where the $(3,2)$ NTM saturates at about $10 \%$ of the minor plasma radius due to the bootstrap drive (section 4 ). We then focus our work on the RF stabilization efficiency issue, taking into account current drive and heating contributions, in both continuous and modulated control schemes (section 5). The dynamics of the island decay is fundamentally different for current or heating applications, with a rapid and large overshoot due to the heating effect, while the island responds slowly to the RF current. The numbers obtained from numerical simulations show a degraded efficiency of the current drive contribution for continuous application, a reduction that vanishes for modulated injection. We document this degradation in section 6 , but we acknowledge that this issue is not fully understood for the moment. The heating contribution is found to follow theoretical predictions, including the background plasma heating effect already studied in the context of classical tearing modes [Maget et al., 2018b]. In conditions comparable to the ITER ones, where the ratio between the applied RF power and the power injected inside the resonant surface ranges between 10 and 20\%, RF heating is expected to contribute to $20 \%$ of the stabilization efficiency in the case of continuous application, a number that appears to be relatively large, but the reason comes from the degraded contribution of the current drive. In the modulated scheme, the net effect of the RF heating decays with time but the overall stabilization efficiency is in accordance with theory as shown in section 5.2. The results are summarized in the conclusion (section 7). 


\section{ECCD-ECRH Criteria for NTM Control}

The dynamics of a magnetic island can be represented in a simplified form with a generalization of the Rutherford Equation [Rutherford, 1973]. We will consider for the present purpose a linear drive that is negative (the island is supposed to be linearly stable), a nonlinear drive due to the bootstrap current perturbation, the RF stabilization terms coming from current drive and heating, as well as a stabilization term due to the background plasma heating.

The magnetic island size $w$ is normalized to the minor plasma radius $a$ and is noted $W=w / a$, and the Generalized Rutherford Equation writes:

$$
\frac{d W}{d \tau_{\eta}}=a \Delta^{\prime}+a \Delta_{b s}^{\prime}+a \Delta_{R F}^{\prime}+a \Delta_{\Omega}^{\prime}\left(P_{r e s}\right)
$$

where $\tau_{\eta}=t /\left(0.82 \tau_{R}\right), \tau_{R}=\mu_{0} a^{2} / \eta$ is the resistive timescale, $a \Delta^{\prime}$ and $a \Delta_{b s}^{\prime}$ are the tearing [Furth et al., 1963] and bootstrap [Fitzpatrick, 1995] terms. The term $a \Delta_{R F}^{\prime}$ represents the effect of the $\mathrm{RF}$ control source, and the last term $a \Delta_{\Omega}^{\prime}\left(P_{\text {res }}\right)$ in (1) represents the stabilization mechanism due to the plasma background heating in the absence of the RF source [Maget et al., 2018b]. The RF term has contributions from the RF current drive and power:

$$
a \Delta_{R F}^{\prime}=a \Delta_{R F}^{\prime C D}+a \Delta_{R F}^{\prime H}\left(P_{R F}, P_{r e s}\right),
$$

where $a \Delta_{R F}^{\prime C D}$ represents the RF-current stabilizing term [Hegna and Callen, 1997] and $a \Delta_{R F}^{\prime H}\left(P_{R F}, P_{r e s}\right)$ is the RF-heating stabilisation term with $P_{R F}$ the RF power deposited inside the island and $P_{\text {res }}$ the residual heating coming from the background plasma heat source [Maget et al., 2018a].

The parameters $\Delta^{\prime}$ in (1) have the following form

$$
\begin{aligned}
a \Delta_{b s}^{\prime} & =6.35 J_{b s} \frac{q}{s} \frac{W}{W^{2}+W_{m}^{2}}, \\
a \Delta_{R F}^{\prime C D} & =-32 \frac{q}{s} J_{R F} \frac{\delta_{R F}}{W^{2}} \eta_{R F} \\
a \Delta_{R F}^{\prime H}\left(P_{R F}, P_{r e s}\right) & =-\alpha_{2}(2 \pi)^{2} C_{\Omega}\left(\mu_{c}, \sigma\right) \frac{a}{L_{T}} \frac{q}{s} J_{\Omega}\left[\left(\frac{P_{R F}+\alpha_{1} P_{r e s}}{P_{e q}}\right)^{1 / \sigma}-\left(\frac{\alpha_{1} P_{r e s}}{P_{e q}}\right)^{1 / \sigma}\right] 5, \\
a \Delta_{\Omega}^{\prime}\left(P_{\text {res }}\right) & =-\alpha_{3}(2 \pi)^{2} C_{\Omega}(1, \sigma) \frac{a}{L_{T}} \frac{q}{s} J_{\Omega}\left(\frac{P_{r e s}}{P_{\text {eq }}}\right)^{1 / \sigma}
\end{aligned}
$$

where $J_{b s}=j_{b s} \mu_{0} R_{0} / B_{0}$ and $J_{\Omega}=j_{\Omega} \mu_{0} R / B_{0}$ are the normalized local bootstrap and ohmic currents, $\delta_{R F}$ is the width of the RF-current density profile normalized to the minor radius and $J_{R F}=j_{R F} \mu_{0} R_{0} / B_{0}$ its peak value. The width $W_{m}$ characterizes the threshold below which heat transport restores the bootstrap current inside the island [Fitzpatrick, 1995]. The temperature gradient length at the resonance is $L_{T}=$ $-T_{s} / d T_{s} / d r$ with $T_{s}$ the temperature at the resonant surface. We also introduce the normalization of the RF deposition width $\mu_{c}=\left(\delta_{R F} / W\right)^{2}$. The safety factor at the resonant surface is $q$ and $s=(r / q) d q / d r$ is the magnetic shear. While $P_{\text {res }}$ represents 
the amount of residual heat deposited inside the whole island by the background plasma heat source, $P_{e q}$ is the background plasma power injected inside the island position, and it is related to the local temperature gradient through the plasma diffusivity [Maget et al., 2018a]. The ratio of these two quantities is instrumental for assessing the impact of local heat sources in a stiff plasma: a large residual heat source contributes to lower the island saturation (equation 6) but it also damps severely the capability to control it with the local heat source provided by the RF system (equation 5). The coefficients $\alpha_{1} \approx 0.1, \alpha_{2} \approx 0.8$ and $\alpha_{3} \approx 1$ have been determined numerically and apply for a continuous RF injection [Maget et al., 2018b]. The function $C_{\Omega}\left(\mu_{c}, \sigma\right)$ is approximated by the formula [Maget et al., 2018a]

$$
C_{\Omega}\left(\mu_{c}, \sigma\right) \approx \frac{3}{4 \pi^{2}}\left[0.8+\frac{0.6}{\sigma}-1.09 \frac{\mu_{c}}{\sigma}+0.242\left(\frac{\mu_{c}}{\sigma}\right)^{2}-0.228 \frac{\mu_{c}}{\sigma} \ln \left(\frac{\mu_{c}}{\sigma}\right)\right]
$$

where $\sigma$ is the transport stiffness. This stiffness parameter would be unity if turbulent transport was independent from the local temperature gradient, but it is large in realistic situations, approaching $\sigma \approx 8$ following turbulent transport simulations for ITER [Kinsey et al., 2011]. In the following we will detail how the terms relative to heat sources, either residual or RF driven, impact the island saturation and the RF specifications that are required to stabilize it.

\subsection{NTM Saturation Size}

We first consider the effect of the residual heat source on the NTM saturation, in the absence of RF control. The residual heat $P_{\text {res }}$ within the NTM due to the plasma background heating is given by the formula [Maget et al., 2018b]

$$
P_{\text {res }}=8 \pi a W \mathcal{J} H_{e q},
$$

with $\mathcal{J}$ the Jacobian at the resonant surface and $H_{e q}$ the heat power density source value at the resonance. The residual heat is distributed over the whole NTM which implies $\mu_{c}=1$ in (7). At saturation, we assume that the characteristic transport width $W_{m}$ is sufficiently small compared to the island size $W_{s}$ that we can neglect it. One has therefore for the saturation without the residual heat source $a \Delta^{\prime} W_{s}=-6.35 J_{b s} q / s$, and after including the residual heat source the island saturates at $W_{s, H}$ with:

$$
1-\frac{W_{s}}{W_{s, H}}+6.22 \alpha_{3} C_{\Omega}(1, \sigma) \frac{a}{L_{T}} \frac{J_{\Omega}}{J_{b s}}\left(\frac{P_{r e s}\left(W_{s, H}\right)}{P_{e q}}\right)^{1 / \sigma} W_{s}=0
$$

Setting $W_{s, H}=W_{s}-\delta$ with $\delta \ll 1$ gives

$$
\delta \approx 6.22 \alpha_{3} C_{\Omega}(1, \sigma) \frac{a}{L_{T}} \frac{J_{\Omega}}{J_{b s}}\left(\frac{P_{r e s}}{P_{e q}}\right)^{1 / \sigma} W_{s}^{2}
$$

The reduction of the island saturation due to the residual heating is therefore an increasing function of the heat power density source value at the resonance, and it is particularly pronounced in a stiff plasma since we have always $P_{r e s} / P_{e q}<1$. This effect is present already for classical islands [Maget et al., 2018b], and numerical simulations will show its evidence on NTM saturation in section 4 . 
The stabilizing influence reported here opposes other effects of the reduced heat transport inside the island. Indeed, the characteristic transport width $W_{m}$ is also reduced due to the larger ratio $\chi_{\|} / \chi_{\perp}$, leading to a larger contribution of the bootstrap term when the island size is close to $W_{m}$ [Fitzpatrick, 2017, Bardòczi et al., 2017]. For an island width much above $W_{m}$, as is expected in the ITER case, this destabilizing effect should not affect strongly the saturated size, in contrast with the effect of the residual heating that scales as $W_{s}^{2}$.

\subsection{RF-Current and-Heating Threshold}

From Equation (1), a criterion on the RF current required to fully stabilize a NTM can be derived [Poli et al., 2015]. The stabilizing effect of the residual heating is participating in the saturation of the island, and is therefore integrated in the $a \Delta^{\prime}$ term that balances, in the absence of RF control, the bootstrap drive. But the damping of the RF heating stabilization due to this residual heating needs to be taken into account. The full stabilization of the NTM requires that the equation

$$
\frac{1}{a \Delta^{\prime}} \frac{d W}{d \tau}=1+\frac{\Delta_{b s}^{\prime}}{\Delta^{\prime}}+\frac{\Delta_{R F}^{\prime}}{\Delta^{\prime}}
$$

is negative for all $W$. We consider different limits of (11) to determine the stabilization conditions related to RF-current and heating deposition as well as the residual heat within the island.

2.2.1. Combinaison of RF current and heating The ECCD-ECRH combination results in a new criterion for the minimal RF-current required to stabilize an NTM. Neglecting residual heat, the criterion is

$$
\left[1+6.22 \alpha_{2} W_{s} \frac{a}{L_{T}} \frac{J_{\Omega}}{J_{b s}} C_{\Omega}\left(\mu_{c}, \sigma\right)\left(\frac{P_{R F}}{P_{e q}}\right)^{1 / \sigma}\right] \frac{\delta_{R F}}{W_{s}} \frac{J_{R F}}{J_{b s}} \eta_{R F} \geq \frac{1}{20.16} .
$$

The RF current required to stabilize the NTM is therefore reduced thanks to the contribution of RF heating. Note that the residual heat $P_{\text {res }}$ does not degrade the stabilization efficiency due to the RF-heating in the case of a non-stiff plasma $(\sigma=1)$.

If we know take into consideration the residual heating, we get a new criterion

$$
\begin{aligned}
& {\left[1+\mathcal{F}\left(P_{R F}, P_{\text {res }}\right)\right] \frac{\delta_{R F}}{W_{s}} \frac{J_{R F}}{J_{b s}} \eta_{R F} \geq \frac{1}{20.16}} \\
& \mathcal{F}\left(P_{R F}, P_{r e s}\right)=6.22 \alpha_{2} W_{s} \frac{a}{L_{T}} \frac{J_{\Omega}}{J_{b s}} C_{\Omega}\left(\mu_{c}, \sigma\right)\left[\left(\frac{P_{R F}+\alpha_{1} P_{r e s}}{P_{e q}}\right)^{1 / \sigma}-\left(\frac{\alpha_{1} P_{r e s}}{P_{e q}}\right)^{1 / \sigma}\right]
\end{aligned}
$$

where the the contribution of RF heating in the NTM stabilization can be significantly reduced, as we will see in the numerical application.

2.2.2. Application to ITER In order to determine the minimum $P_{R F}$ required to stabilize an NTM located at the resonant surface $q=3 / 2$ for ITER, we use the numerical values of $J_{\Omega} / J_{b s}=2.3$, and $a / L_{T}=2.6$ at the resonant surface $q=3 / 2(\sqrt{\psi}=0.76$ 
or $\sqrt{\Phi}=0.64$ in terms of toroidal flux co-ordinate) from the ITER-like equilibrium described in section 3.2. The magnetic shear at $q=3 / 2$ is $s \approx 1.3$. For the RF specifications, we assume an available power of $13.3 \mathrm{MW}$ and a toroidal injection angle of 20 degrees that provides a current drive efficiency $\eta_{C D}$ of about $9 \mathrm{kA} / \mathrm{MW}$ and gives $I_{R F} \approx 120 \mathrm{kA}$ [Farina et al., 2014, Figini et al., 2015]. For the nominal ITER scenario, where the plasma current is $I_{p}=15 M A$ and the power injected inside $q=3 / 2$ is about $P_{e q}=90 M W$ [Casper et al., 2014], we get therefore $I_{R F} / I_{p}=0.8 \%$ and $P_{R F} / P_{e q}=0.15$ (see section 3). In addition we take $\delta_{R F}=0.017$ (i.e. $3.4 \mathrm{~cm}$ in ITER where the minor radius is $a=2 \mathrm{~m}$ ), a value that corresponds to the nominal value of the EC system specification [Figini et al., 2015] (note that $\delta_{R F}$ is defined as the full width at half the peak value of the RF current deposition profile). This gives $J_{R F} / J_{b s}=1.26$ at $q=3 / 2$. Note that the profile broadening issue [Peysson et al., 2011, Decker, J. et al., 2012, Sysoeva et al., 2015, Snicker et al., 2018] does not affect the product $\delta_{R F} J_{R F}$, but it does enter in the problem via the $\mathrm{RF}$ stabilization efficiency $\eta_{R F}$ that depends on $W / \delta_{R F}$. For the island saturation size, we take $W_{s}=0.1$ (see section 4 ). The residual heating for this island size is evaluated around $P_{r e s} / P_{e q}=0.08$ (see section 3.2), a value that is therefore about twice lower than the RF power contribution. Finally we approximate the RF-current efficiency $\eta_{R F}$ by the fits given in Sauter et al. for continuous and $50 \%$ modulated RF application [Sauter, 2004]

$$
\begin{aligned}
\eta_{R F}^{C W}\left(\frac{W}{W_{R F}}\right) & =\frac{1}{4} \frac{\left(\frac{W}{W_{R F}}\right)^{2}}{1+\frac{2}{3}\left(\frac{W}{W_{R F}}\right)^{2}} \\
\eta_{R F}^{\text {mod }}\left(\frac{W}{W_{R F}}\right) & =\frac{9}{20} \tanh \left(\frac{2}{5} \frac{W}{W_{R F}}\right)
\end{aligned}
$$

with $W_{R F}=\sqrt{\ln 2} \delta_{R F}$. The RF current and heating that are required to fully stabilize the $(3,2)$ NTM are computed by increasing $J_{R F}$ and $P_{R F}$ until equation 11 has no positive value. On the question of the critical width $W_{m}$, we compare two different approaches, one where we assume $W_{m}=0$ and $\eta_{R F}\left(\frac{W_{s}}{\delta_{R F}}\right) \approx 0.35$, and the other where we take $W_{m}=2 \%$ and the expression of equation 15 for the RF efficiency. It is known indeed that the RF current requirement decreases as $W_{m}$ is increased, and we find that the first option gives conservative constraints on this requirement, as shown below.

Figure 1 represents the values of the RF-current and -heating necessary to stabilize the $(3,2)$ NTM with or without residual heating. Values under the lines are unable to stabilize the $(3,2) \mathrm{NTM}$, and the ITER reference is indicated by the vertical line at $P_{R F} / P_{e q} \approx 0.15$. The requirement in terms of RF current in the absence of RF heating is $J_{R F} / J_{b s} \sim 0.74$ (for $W_{m}=2 \%$ ). The gain that can be expected in ITER from the heating contribution is negligible in a non-stiff plasma, and is, for $\sigma=8$, around $11 \%$ in the absence of residual heating. With the expected value $P_{r e s} / P_{e q} \approx 0.08$, the contribution of RF heating to the NTM stabilization translates into a moderate reduction of the RF current requirement of only $4 \%$. The value of about $J_{R F} / J_{b s}=1.26$ appears therefore 


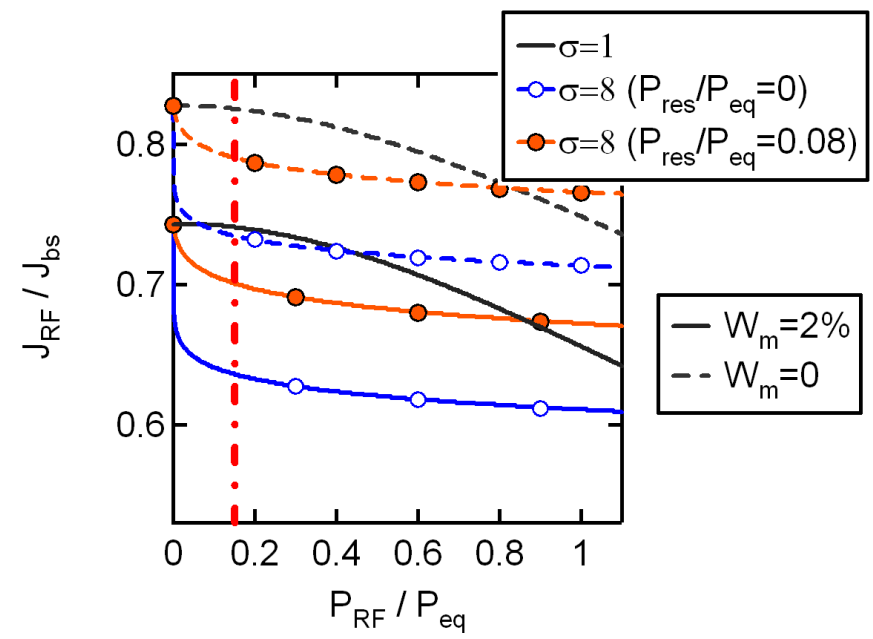

Figure 1. Required EC current density to stabilize an NTM as given by (13) as a function of the RF power $\left(P_{R F}\right)$ for a non-stiff plasma $(\sigma=1)$ and a plasma with stiffness parameter $\sigma=8$, with a residual heating $P_{r e s}=0$ or, as estimated in ITER, $P_{\text {res }} / P_{e q}=0.08$. Dashed lines correspond to the hypothesis $W_{m}=0$ and $\eta_{R F}=0.35$ and full lines to $W_{m}=2 \%$ and $\eta_{R F}(W)$. The vertical line shows the expected value in ITER, $P_{R F} / P_{e q} \approx 0.15$.

to be sufficient for the stabilization of the $(3,2)$ NTM in ITER, with a margin of about a factor of two, and the heating has a negligible contribution. For a broader application range, e.g. in present tokamaks where the ratio $P_{R F} / P_{e q}$ can be of order unity, the figure illustrates how the plasma stiffness reduces the advantage using a large RF power. It should be kept in mind, however, that the equilibrium effect on the current profile, not taken into account here, can be significant (and stabilizing if well aligned with the resonance) in such cases [Glasser et al., 1977, De Luca et al., 1986, Westerhof, 1987, Sing et al., 1993, Pletzer and Perkins, 1999, Kislov and T-10 Team, 2001].

The requirement on the EC system expressed in equation 12 can also be written in terms of the current drive efficiency $\eta_{C D} \equiv I_{R F} / P_{R F}$. For an elongated plasma, we use the approximation $I_{R F} \approx \pi^{3 / 2} / \sqrt{2 \ln 2} x_{s} \sqrt{1+\kappa^{2}} \delta_{R F} a^{2} j_{R F}$ with $\kappa \approx 1.8$ the plasma elongation, in order to derive a simple criterion. The minimum required power to stabilize the NTM can then be expressed as

$$
\left[1+\mathcal{F}\left(P_{R F}, P_{r e s}\right)\right] \frac{P_{R F}}{P_{e q}} \eta_{R F} \frac{\sqrt{2 \ln 2} P_{e q} \eta_{C D}}{\pi^{3 / 2} x_{s} \sqrt{1+\kappa^{2}} a^{2} W_{s}} \frac{\mu_{0} R_{0}}{J_{b s} B_{0}} \geq \frac{1}{20.16}
$$

where $x_{s}=r_{s} / a$. Figure 2 depicts (17) where the red square corresponds to $\eta_{C D} \approx 9 \mathrm{kA} / \mathrm{MW}$, which is the ITER value at $q=3 / 2$ that will be used in the numerical simulations. The contribution of heating in the stabilization can be isolated by computing $\left(P_{R F}^{C D}\right.$ only $\left.-P_{R F}\right) / P_{R F}$, as shown in figure 3 . This contribution decreases naturally at larger $\mathrm{CD}$ efficiency, and since the power requirement is lower with $P_{R F} / P_{e q}$ getting low as well, the plasma stiffness tends to enhance this contribution. At low CD efficiency, either intentional by directing the RF antenna more perpendicularly to the 


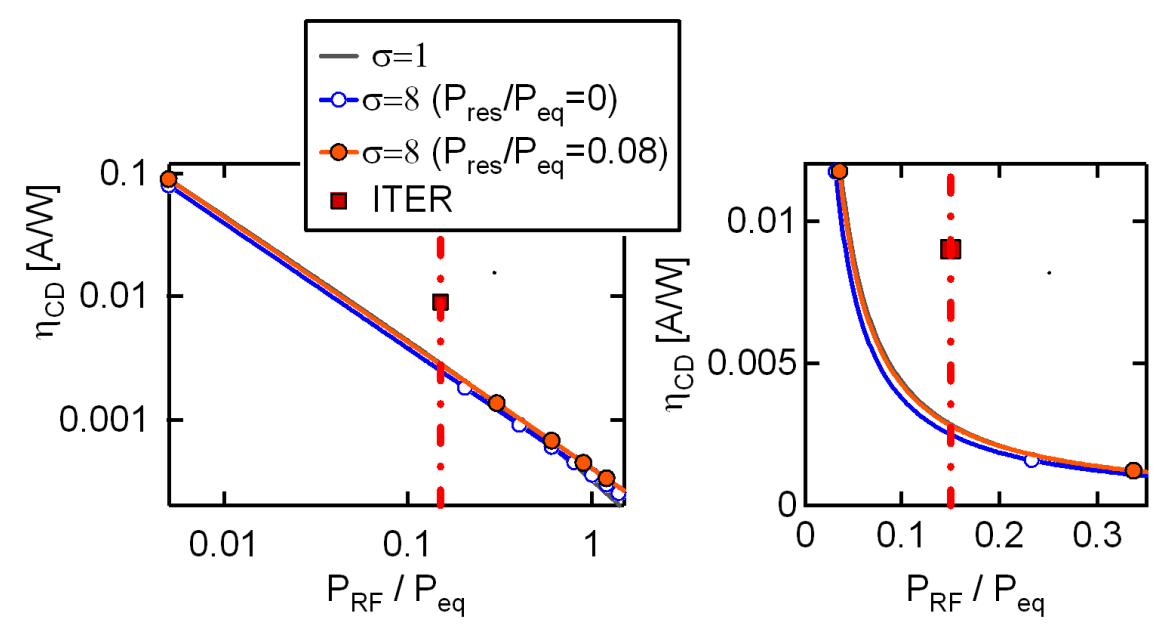

Figure 2. Current drive efficiency $\eta_{C D} \equiv I_{R F} / P_{R F}$ required for $(3,2)$ NTM stabilization as a function of the RF power $\left(P_{R F} / P_{e q}\right)$, with $\sigma$ the stiffness strength, including a residual heat source $P_{r e s} / P_{e q}=0.08$ (left: $\log$ scale, right: linear scale), and we use $W_{m}=2 \%$ and $\eta_{R F}(W)$. The ITER reference used in the numerical simulations is marked with a red square.

magnetic surface or due to a low plasma temperature, the power required to stabilize the NTM becomes large and the ratio $P_{R F} / P_{e q}$ rapidly exceeds the value where plasma stiffness is favorable. In ITER, with the parameters detailed earlier and taking into account a residual heating of about $8 \%$, the reduction of the required RF power due to the heating contribution does not exceed $4 \%$ of the injected power when we take $\eta_{C D} \approx 9 \mathrm{kA} / \mathrm{MW}$, and at the lowest $\mathrm{CD}$ efficiency allowed within the available power $\eta_{C D} \approx 3 \mathrm{kA} / \mathrm{MW}$ (see figure 2), the heating contribution would not exceed about $10 \%$.

\section{Non-Linear Simulations Set-Up}

\subsection{Equations Solved}

We use the XTOR code [Lütjens and Luciani, 2010] to solve the following non-linear set of normalized MHD equations

$$
\begin{aligned}
\left(\partial_{t}+\mathbf{V}_{i} \cdot \nabla\right) \rho= & -\rho \nabla \cdot \mathbf{V}_{i}-\nabla \cdot \Gamma_{a n}+S \\
\left(\partial_{t}+\mathbf{V} \cdot \nabla\right) p= & -\Gamma p \nabla \cdot \mathbf{V}-d_{i} \Gamma \mathbf{K} \cdot\left[\frac{p}{\rho} \nabla p_{i}+\frac{p_{i}}{\rho} \nabla p_{i}-\frac{p_{e}}{\rho} \nabla p_{e}+\frac{p_{e}^{2}-p_{i}^{2}}{\rho^{2}} \nabla k \rho\right) \\
& +(\Gamma-1)\left(H_{e q}-\nabla \cdot \mathbf{q}_{\chi}+H_{R F}\right), \\
\rho\left(\partial_{t}+\mathbf{V} \cdot \nabla\right) \mathbf{V}= & -\rho \mathbf{V}_{i}^{\star} \cdot \nabla \mathbf{V}_{\perp}+\mathbf{J} \times \mathbf{B}-\nabla p+\nabla \cdot \nu \nabla \mathbf{V}_{i}, \\
\partial_{t} \mathbf{B} & -\nabla \times \mathbf{E},
\end{aligned}
$$

with

$$
\mathbf{E}+\mathbf{V} \times \mathbf{B}=\eta\left[\mathbf{J}-\mathbf{J}_{b s}-\mathbf{J}_{C D}-\mathbf{J}_{R F}\right]-d_{i} \frac{\nabla_{\|} p_{e}}{\rho},
$$




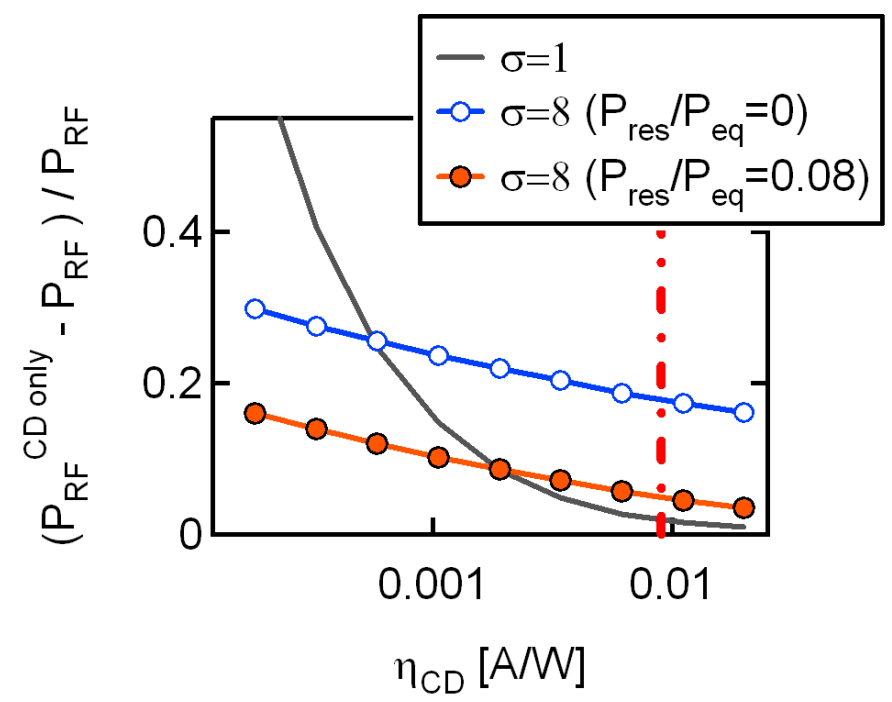

Figure 3. Contribution of RF heating in the stabilization of the $(3,2)$ NTM island as a function of the current drive efficiency $\eta_{C D} \equiv I_{R F} / P_{R F}$. The vertical line at $\eta_{C D}=0.009$ is the value taken in the simulations for ITER.

with $\rho=n_{i} / n_{i}(0)$ the normalised mass density on axis, $\mathbf{V}=\mathbf{V}_{\mathbf{E}}+\mathbf{V}_{\|, i}, \mathbf{V}_{\mathbf{E}}=\mathbf{V} \times \mathbf{B} / B^{2}$, $\mathbf{V}_{\|, i}$ the ion parallel velocity, $d_{i}=V_{A} /\left(a \omega_{c i}\right)$ the normalized ion skin depth with $V_{A}=$ $B_{0} / \sqrt{\mu_{0} \rho(0)}$ the Alfvén velocity, $\Gamma=5 / 3$ represents the ratio of specific heat and $H_{e q}=$ $-(\Gamma-1) \nabla \cdot \chi_{\perp}^{0} \nabla_{\perp} p(t=0)$ the heat source. The symbol $H_{R F}$ represents the externally driven heat $(\mathrm{ECRH})$. The diffusive heat flux is $\mathbf{q}_{\chi}=-\rho \chi_{\|} \mathbf{b}(\mathbf{b} \cdot \nabla T)-\rho \chi_{\perp} \nabla_{\perp} T$. Here $\mathbf{b}=\mathbf{B} /|\mathbf{B}|, T=p / \rho$ and $\chi_{\perp}$ represents the perpendicular diffusivity due to turbulent transport processes as [Maget et al., 2018a]

$$
\chi_{\perp}=\chi_{\perp}^{0}\left|\frac{T^{\prime}}{T_{e q}^{\prime}}\right|^{\sigma-1},
$$

where $\chi_{\perp}^{0}$ and $T_{e q}$ are the heat diffusivity and the temperature equilibrium in the absence of NTM, $\sigma$ the stiffness parameter and $T$ the temperature. The ' denotes derivatives in the radial direction. In ITER, a typical value of the stiffness strength at the $q=3 / 2$ resonant surface is $\sigma=8$ [Kinsey et al., 2011]. The particle source $S$ restores the mass density profile, $\Gamma_{a n}=\left(-D_{\perp} \nabla \rho+\rho \mathbf{V}_{\text {pin }}\right)$ is the anomalous particle flux modeling the turbulent particle transport, $D_{\perp}=2 \chi_{\perp} / 3$ the perpendicular diffusion coefficient and $\mathbf{V}_{\text {pin }}$ a pinch velocity that restores the density profile in the core region $(\sqrt{\psi} \in[0,0.9])$ where the density source is zero, as described in [Maget et al., 2016]. Both the heat source and particle sources are defined by their equilibrium profiles (section 3.2). We use for the bootstrap current the ad-hoc formulation $\mathbf{J}_{\mathbf{b s}}=J_{b s}^{e q}|\nabla p| /\left|\nabla p_{e q}\right| \mathbf{b}$ where the initial equilibrium bootstrap is computed within the CHEASE code [Lütjens et al., 1996]. The non-inductive current density source $\mathbf{J}_{\mathbf{C D}}=J_{C D} \mathbf{e}_{\varphi}$ is then prescribed as $J_{C D}=\left(J_{\varphi}-J_{b s, \varphi}-E_{0} / \eta\right)_{t=0}$ with $E_{0}$ a constant prescribed at the edge such that $E_{0} /\left(\eta(0) J_{\varphi}(0)\right)=0.75$ (see Février et al [Février et al., 2018]), i.e. the ohmic current 
represents $75 \%$ of the total current at the plasma center, and about $54 \%$ of it at $q=3 / 2$.

The ECCD and ECRH sources are evolved by the following governing equations

$$
\begin{aligned}
& \partial_{t} J_{R F}=\nu_{f}\left(J_{s}-J_{R F}\right)+\chi_{\perp}^{R F}|B| \nabla^{2} \frac{J_{R F}}{|B|}+\chi_{\|}^{R F}|B| \nabla_{\|}^{2} \frac{J_{R F}}{|B|}, \\
& \partial_{t} H_{R F}=\nu_{f}\left(H_{s}^{R F}-H_{R F}\right)+\chi_{\perp}^{R F} \nabla^{2} H_{R F}+\chi_{\|}^{R F} \nabla_{\|}^{2} H_{R F},
\end{aligned}
$$

with $\mathbf{J}_{R F}=J_{R F} \mathbf{b}$ and $\nu_{f}=\nu_{e i}\left(v_{t h} / v_{r e s}\right)^{3}$ the collision frequency of fast electrons for which we use $v_{t h} / v_{\text {res }}=1 / 2$. The evolution equation for the EC current has been slightly modified compared with Février et al [Février et al., 2016] so that we have, in the limit $\chi_{\|}^{R F} \rightarrow \infty$, a divergence-free RF current. The subscript $s$ denotes the source terms that are implemented as 1D or 3D Gaussian

$$
A_{R F}^{S}(y, \theta \phi)=A_{0}^{S} \exp \left(-\frac{\left(y-y_{R F}^{0}\right)^{2}}{2 \sigma_{r}^{2}}-\frac{\left(\theta-\theta_{R F}^{0}\right)^{2}}{2 \sigma_{\theta}^{2}}-\frac{\left(\phi-\phi_{R F}^{0}\right)^{2}}{2 \sigma_{\phi}^{2}}\right),
$$

where $A \equiv H$ or $J$ and $y \equiv \sqrt{\psi}$. The source width $\delta_{R F}=2 \sqrt{2 \ln 2} \sigma_{r}$ is the full width at half maximum of the source.

\subsection{Equilibrium}

We use a simplified version of the ITER plasma shape given in Casper et al [Casper et al., 2014] using an up-down symmetric equilibrium (see figure 4). The inverse aspect ratio is $\epsilon=a / R_{0}=0.323$ for $a$ the minor radius and $R_{0}=6.35 \mathrm{~m}$ the major radius at the geometric axis. Other parameters at the geometric axis are the magnetic field, pressure, mass density and electronic temperature given as $B_{0}=5.17 \mathrm{~T}$, $p(0)=691.5 \mathrm{kPa}, N_{i}(0)=10^{20} \mathrm{~m}^{-3}$ and $T_{e}(0)=21.3 \mathrm{keV}$ respectively with $T_{i} / T_{e}=1$. The normalized density profile is parametrized as

$$
\rho=\frac{1-d_{1} y^{d_{2}}}{1+d_{3} y^{d_{4}}}
$$

with $\left[d_{1}, d_{2}, d_{3}, d_{4}\right]=[0.3,7,1.2,60]$, which gives a flat density profile in the core.

To determine our initial profiles, we solve the Grad-Shafranov equation using the Chease code [Lütjens et al., 1996] with the following analytical equilibria

$$
\begin{aligned}
& \partial_{\psi} p=-0.61+0.087\left(1+\tanh \left(\frac{y-0.6}{0.3}\right)\right), \\
& F \partial_{\psi} F=0.696\left(\tanh \left(\frac{y-0.67}{0.18}\right)-1\right),
\end{aligned}
$$

with the flux function $F=R B_{\phi}, y=\sqrt{\psi}$ and $\psi$ the normalized poloidal flux. In our simulations, the equilibrium profiles determined by solving (29) are qualitatively similar with the $\mathrm{Q}=10$ ITER baseline inductive scenario [Casper et al., 2014], where the pressure pedestal has been ignored. The safety factor, current density and bootstrap current density, and pressure profiles are shown in figure 5. The position of the $q=3 / 2$ surface is at $\sqrt{\psi}=0.761$, or in terms of normalized toroidal flux coordinate $\sqrt{\Phi}=0.643$, while the $q=2$ surface is at $\sqrt{\psi}=0.868(\sqrt{\Phi}=0.773)$, values that are consistent with the ITER scenario specifications [Figini et al., 2015]. 

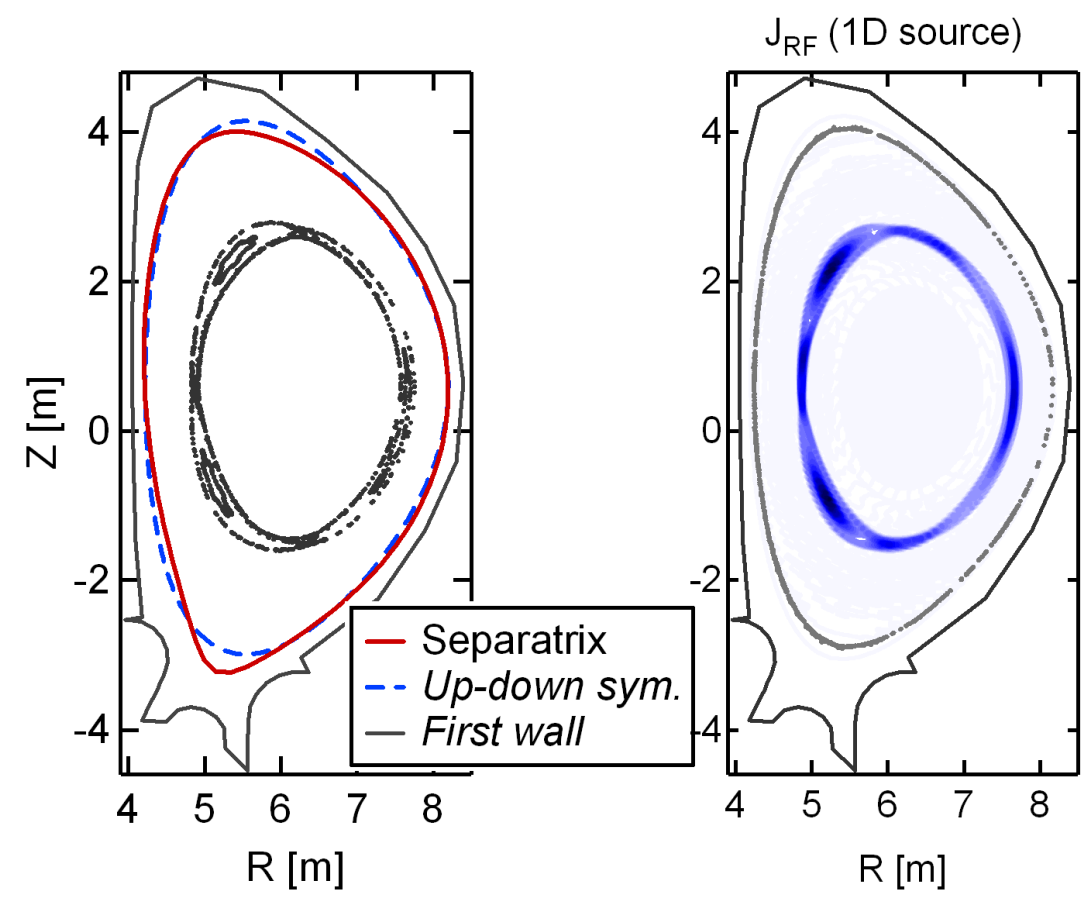

Figure 4. Left: plasma shape used for the simulations (up-down symmetry), with original asymmetric shape. The Poincaré plot of the $(3,2)$ island at saturation is also shown. Right: contour plot of the RF current density for a $1 \mathrm{D}$ source injected at the saturated island position.

In the simulations, we take the ratio of parallel to perpendicular heat diffusivity as $\chi_{\|} / \chi_{\perp}(0)=10^{8}$ together with $S_{0} \chi_{\perp}=150$. Here $S_{0}=\tau_{R} / \tau_{A}$ is the Lundquist number at the plasma center, $\tau_{A}=R_{0} \sqrt{\mu_{0} \rho(0)} / B_{0}$ the Alfvén time, $\tau_{R}=\mu_{0} a^{2} / \eta(0)$ the resistive time scale and $\eta(0)$ the resistivity at the plasma center. This leads at $q=3 / 2$ to a characteristic transport width $W_{\chi} \approx 3.3 \%$ and $W_{m}=1.8 W_{\chi} \approx 6 \%$ at equilibrium. This is larger than the expected value in ITER, due to numerical limitations on the value of $\chi_{\|}$, but still significantly lower than the saturated width. For the figures that will be shown, we rescale the time scale of the simulations with respect to the resistive time as

$$
t=\tau_{A} \frac{S^{\text {real }}}{S_{0}} \bar{t}
$$

where $S^{\text {real }} \sim 2 \times 10^{10}$ and $S_{0}=10^{7}$ are the typical Lundquist number in the core of ITER and in the simulations respectively.

\subsection{Background heat source}

As described in section 2, the plasma background heating has a significant effect on the island saturation size when plasma stiffness is large, and it also reduces the ECRH capability in controlling the NTM. The default heat source used in XTOR derives from the assumption of an initial diffusivity coefficient that follows plasma resistivity, and 


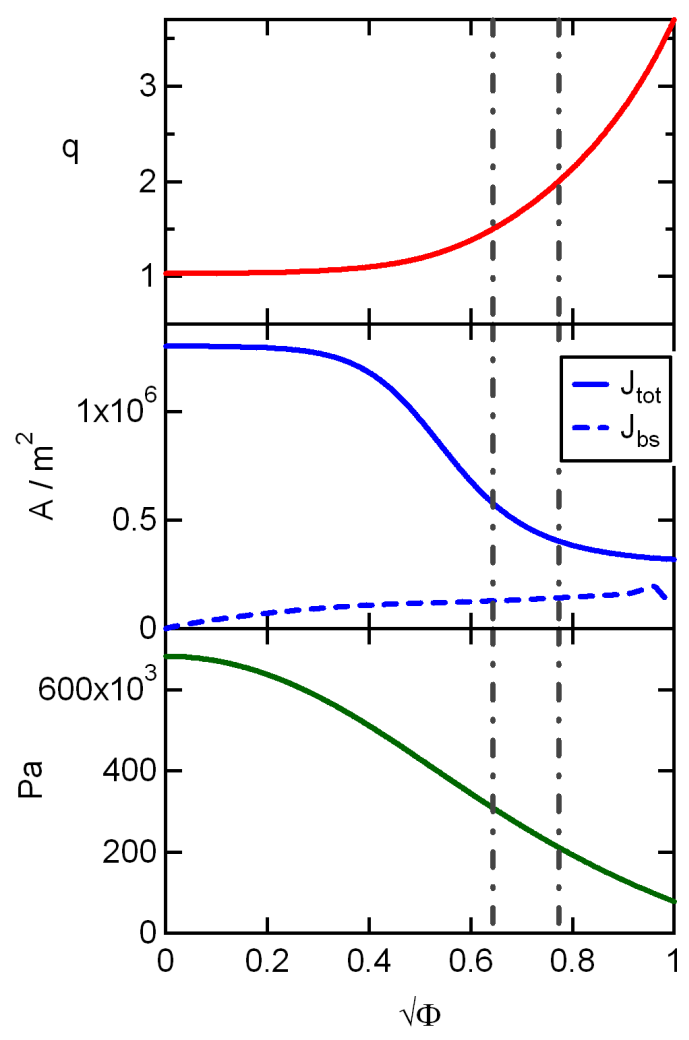

Figure 5. Safety factor, current density, bootstrap current density and pressure profiles used in the simulations. The vertical lines indicates the positions of $q=3 / 2$ and $q=2$.

increases therefore from the core to the edge. Consequently, we need to compute a modified heat source that has an ITER-like radial shape, peaking at the plasma center, for cases with $\sigma>1$. A good fit of the power density in Casper et al. [Casper et al., 2014] is $H_{e q}^{I T E R} \sim(1-\sqrt{\psi})^{3 / 2}$. This is very close, although slightly broader, to the heat source produced by fusion reaction $H_{\text {fus }} \propto p^{2}$. We force the ITER-like heat source to be equal to the default heat source outside $\sqrt{\psi}>0.8$, and the profile inside this radius is scaled so that the total injected power $\left(P_{e q}\right)$ remains unchanged. The radial profiles of the initial and ITER-like heat sources are shown in Figure 6.

Less residual heat is present within the NTM for the ITER-like heat source, with a power density source $H_{e q}$ that is reduced by a factor 5.8 at $q=3 / 2$. Note that the modified heat sources cannot be used with the non-stiff transport model, since it would lead to a very strong modification of the plasma equilibrium, as shown in [Maget et al., 2018b], while the change of temperature gradient due to a variation of the heat source in a stiff plasma remains moderate. 


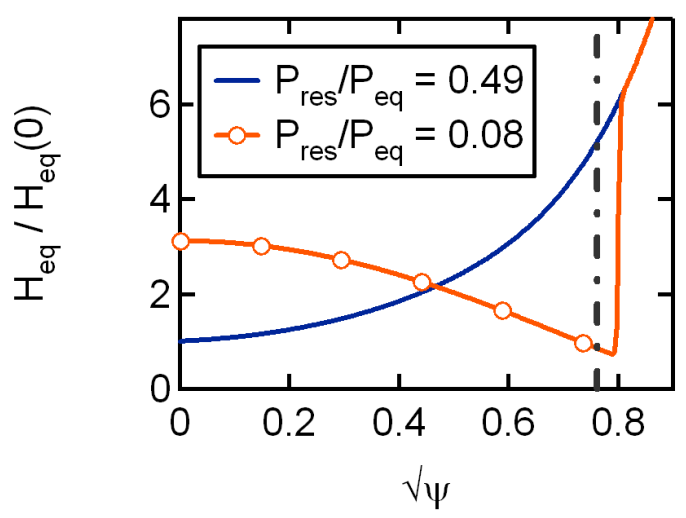

Figure 6. ITER-like heat source equilibrium profile normalized to the default heat source value at the core $(\psi=0)$.

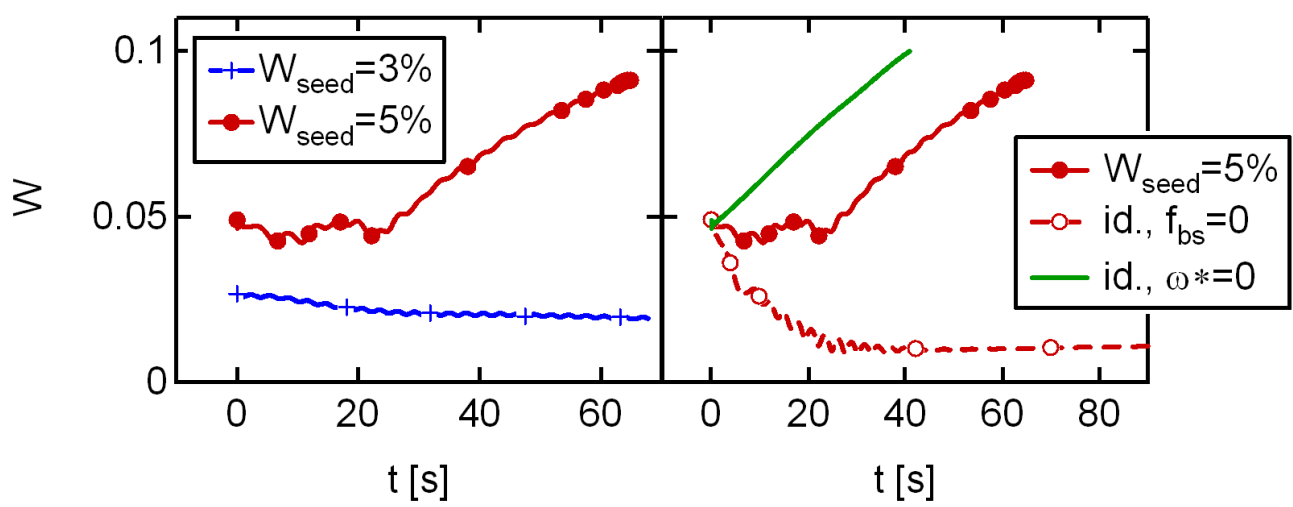

Figure 7. (3,2) NTM time evolution. Left: two different seed sizes, showing the metastable nature of the island. Right: simulation without bootstrap $\left(J_{b s}=0\right)$ current showing that it constitutes the nonlinear drive, and without diamagnetic rotations $\left(\omega^{\star}=0\right)$ showing their stabilizing effect.

\section{4. $(3,2)$ NTM Saturation}

The $n=2$ mode is linearly stable and requires a seed island to access the metastable NTM branch. This is obtained by amplifying the decaying mode structure obtained from a large initial perturbation, and inserting it as an initial condition in the simulation. The spatial discretization is of 512 points in the radial direction, and we use for the saturation issue 96 points in the poloidal direction, and 16 in the toroidal direction. We simulate a half torus, i.e. we retain even toroidal mode numbers only.

Figure 7 depicts the time evolution of the magnetic island size for different seeds and situations. The metastable character of the $(3,2)$ island is evidenced by the fact 
that the island is stable for a seed of $2.6 \%$ of the minor radius, and unstable for a seed of $5.2 \%$. The island does not grow when we artificially set the bootstrap current to zero, showing that the magnetic island is driven by the bootstrap current, and is therefore an NTM. We also test the implication of diamagnetic rotations $\left(\omega^{\star}\right)$ on the island stability. We find that diamagnetic effects have stabilizing properties that delay the onset of the NTM growth. When setting the diamagnetic rotations to zero $\left(\omega^{\star}=0\right)$ by taking $d_{i}=0$ in the MHD equations, the NTM grows immediately after the seed introduction.

The $(3,2)$ NTM is close to saturation after about a minute of growth, and its size is about $10 \%$ of the minor radius, i.e., $w \approx 20 \mathrm{~cm}$. At this stage of the simulation, the poloidal and toroidal discretizations are increased to 128 and 24 points in the poloidal and toroidal directions respectively. We then follow two values of the stiffness parameter, the default one at $\sigma=1$ that corresponds to a non-stiff plasma, and $\sigma=8$ that corresponds to the expected stiffness strength in ITER. For the stiff case, we also compare the default heat source and the ITER-like one, as explained in section 3.3. For the actual island size $W \approx 9.5 \%$, the ratio $P_{r e s} / P_{e q}$ is about $49 \%$ for the default heat source, and about $8 \%$ for the ITER-like one.

The evolution of the island size when using these two values of the residual heat source for $\sigma=8$ is shown in figure 8. As expected from the analytical model, residual heat sources are stabilizing, and the island moves to a lower size. For the case with $P_{r e s} / P_{e q}=49 \%$, the island growth stops at about $9.5 \%$. Using the analytical estimate for the reduction of the saturated width, equation 10 , we find that $\delta \approx 1.6 \%$ for the ITER-like heat source $\left(P_{\text {res }} / P_{e q}=8 \%\right)$ and $\delta \approx 2.0 \%$ for the default heat source $P_{r e s} / P_{e q}=49 \%$, taking for the saturated width $W_{s}=W_{s}\left(P_{r e s} / P_{e q}=\right.$ $49 \%)+\delta\left(P_{\text {res }} / P_{e q}=49 \%\right) \approx 11 \%$ in the formula. We assume here that in this case the saturation is around $9.5 \%$. The residual heat source is therefore expected to contribute to a reduction of about $15 \%$ of the island saturation in ITER. The saturated size of the $(3,2)$ island would be around $w \approx 22 \mathrm{~cm}$, a value is consistent with previous estimates around $25 \mathrm{~cm}$ [Poli et al., 2015]. We do not observe any sign of the destabilizing influence of a lower transport width, as expected theoretically when residual heat is not taken into account [Fitzpatrick, 2017]. This could result from the island size being sufficiently large compared to $W_{m}$, where the destabilization effect tends to vanish. But the amplitude of the residual heating effect might also be sufficiently large to overcome what remains of this destabilization.

\section{5. $(3,2)$ NTM Control}

The $(3,2)$ NTM can be controlled driving RF-current (ECCD) or -heating (ECRH) inside the helical structure of the island. For this study we consider either a 1D RF source, equivalent to a continuous RF application of a 3D source on an island that rotates rapidly with respect to its characteristic evolution time [Ayten and Westerhof, 2012], or a 3D RF source for modulated EC injection. We assume that the RF deposition is radially localized at the position of the O-point $(\sqrt{\psi}=0.755)$, where the stabilization 


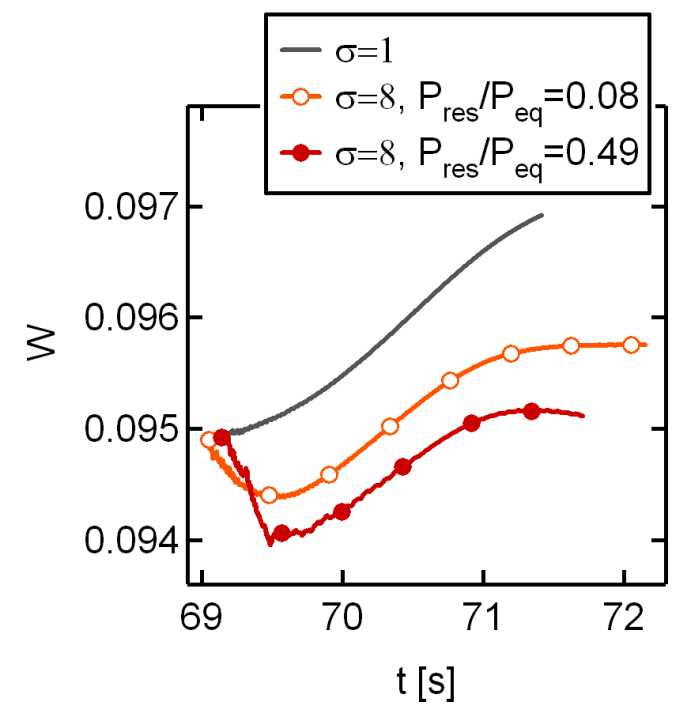

Figure 8. Island width evolution for $\sigma=1,8$ with different residual heat source $\left(P_{\text {res }} / P_{\text {eq }}\right)$.

is the most efficient. We use $\chi_{\|}^{R F}=10^{3}$ and $\chi_{\perp}^{R F}=10^{-7}$ in the RF evolution equations 25 and 26. The RF source width is set at $\sigma_{r}=8.9 \times 10^{-3}$, which gives a theoretical $\delta_{R F}^{t h}=0.02$ in $\sqrt{\psi}$ co-ordinate (and $\delta_{R F}^{t h}=0.017$ in $\sqrt{\Phi}$ co-ordinate that is used in the Rutherford analysis). The broadening caused by diffusion is evaluated in a separate simulation without island. We find an effective width of about $\delta_{R F}^{e f f} \approx 0.023$ in $\sqrt{\Phi}$ co-ordinate.

The role of heating on the NTM decay rate is emphasized by varying the amount of applied ECRH, i.e. by modifying the value of $P_{R F} / P_{e q}$, for different values of the stiffness parameter $\sigma$. The role of the residual heat source in the case of stiff profiles is evidenced by comparing the default and ITER-relevant heat source. Because the time needed for a full stabilization of the $(3,2)$ saturated NTM is beyond reach with the settings that we have chosen (in particular the spatial discretization and $\chi_{\|}$value), we focus our study on the island decay rate that is a crucial element for the RF system requirements, as shown in section 2 .

We will use two different characterizations of the island reduction by RF control. First, we consider the jump in the island size due to the RF-current and -heating application. This jump is represented by

$$
S_{0} d \widetilde{W} / d t
$$

where we isolate the RF contribution by defining

$$
\widetilde{W}=W_{R F}(t)-W_{s}(t)+W_{s}\left(t_{R F}\right) .
$$

Here $t_{R F}$ is the time the RF is applied, $W_{R F}(t)$ is the NTM normalized width once RF control is applied, and $W_{s}(t)$ the island width without RF control. 
The other quantitative parameter is the effective stabilization efficiency $\eta_{R F}^{e f f}$, computed as

$$
\eta_{R F}^{e f f}=-0.82 S \frac{d \widetilde{W}}{d t} \frac{W^{2}}{D_{R F}}
$$

Here $D_{R F}$ reads

$$
D_{R F}=\frac{16}{\pi} \frac{\mu_{0} R_{0} I_{R F}}{s \psi_{s}^{\prime}}
$$

where $\psi_{s}^{\prime}$ is the derivative of the poloidal flux on the resonant surface. Equation (34) measures the efficiency of the applied RF on the $(3,2)$ NTM stabilization, and can be compared with theoretical values [Hegna and Callen, 1997, Sauter, 2004].

In the case of modulation it is also convenient to define an average effective stabilization efficiency $\left\langle\eta_{R F}^{e f f}\right\rangle$ as

$$
\left\langle\eta_{R F}^{e f f}\right\rangle(t)=-\left(\int_{t_{R F}}^{t} d t 0.82 S \frac{d \widetilde{W}}{d t}\right) /\left(\int_{t_{R F}}^{t} d t \frac{D_{R F}}{W^{2}}\right)
$$

\subsection{Continuous ECCD/ECRH Injection}

5.1.1. Overview The contour of the RF current density injected at the island position for a $1 \mathrm{D}$ source is shown in figure 4 . In this case, the current is distributed equally over the island phase. The mains results for $\sigma=1$ and $\sigma=8$ with the two heating sources are summarized in figure 9, with pure current drive stabilization and with the additional contribution of RF heating at moderate levels $P_{R F} / P_{e q}=0.11$ and $P_{R F} / P_{e q}=0.22$, consistent with the operating range of ITER or Asdex-Upgrade. The decay rate and stabilization efficiency are shown with a sliding time average window of $40 \mathrm{~ms}$.

The stabilization due to the RF current is insensitive to the plasma stiffness and reaches in about $100 \mathrm{~ms}$ a steady value $\eta_{R F}^{e f f} \approx 0.11$. This value is significantly lower than the theoretical prediction from equation 15 , which would give $\eta_{R F}^{C W} \approx 0.33$. This degradation by a factor around 3 has been confirmed in separate simulations with simpler geometry and MHD model, so that it seems a robust trend of the code prediction (see the discussion in section 6).

The response of the island to localized heating is significantly different from the pure RF current response. It is characterized by two different characteristic time scales. On a time scale of the order of a fraction of a second, we observe a strong decay rate that manifests the short characteristic time of energy confinement compared with resistive time. This overshoot of the stabilization efficiency is shorter in stiff plasma conditions, thus evidencing the role of the turbulence increase due to the RF heating inside the island. The stabilization efficiency rises to about a factor of 6 above the pure RF current effect. On a longer time scale, the heating effect has a modest contribution to the island decay. Because the scale of the plots in figure 9 is dominated by the fast

response of RF heating, the longer term contribution is not clearly visible, but if we focus on a time window where the decay rate has reach a quasi steady state, we see that it is not completely negligible. 


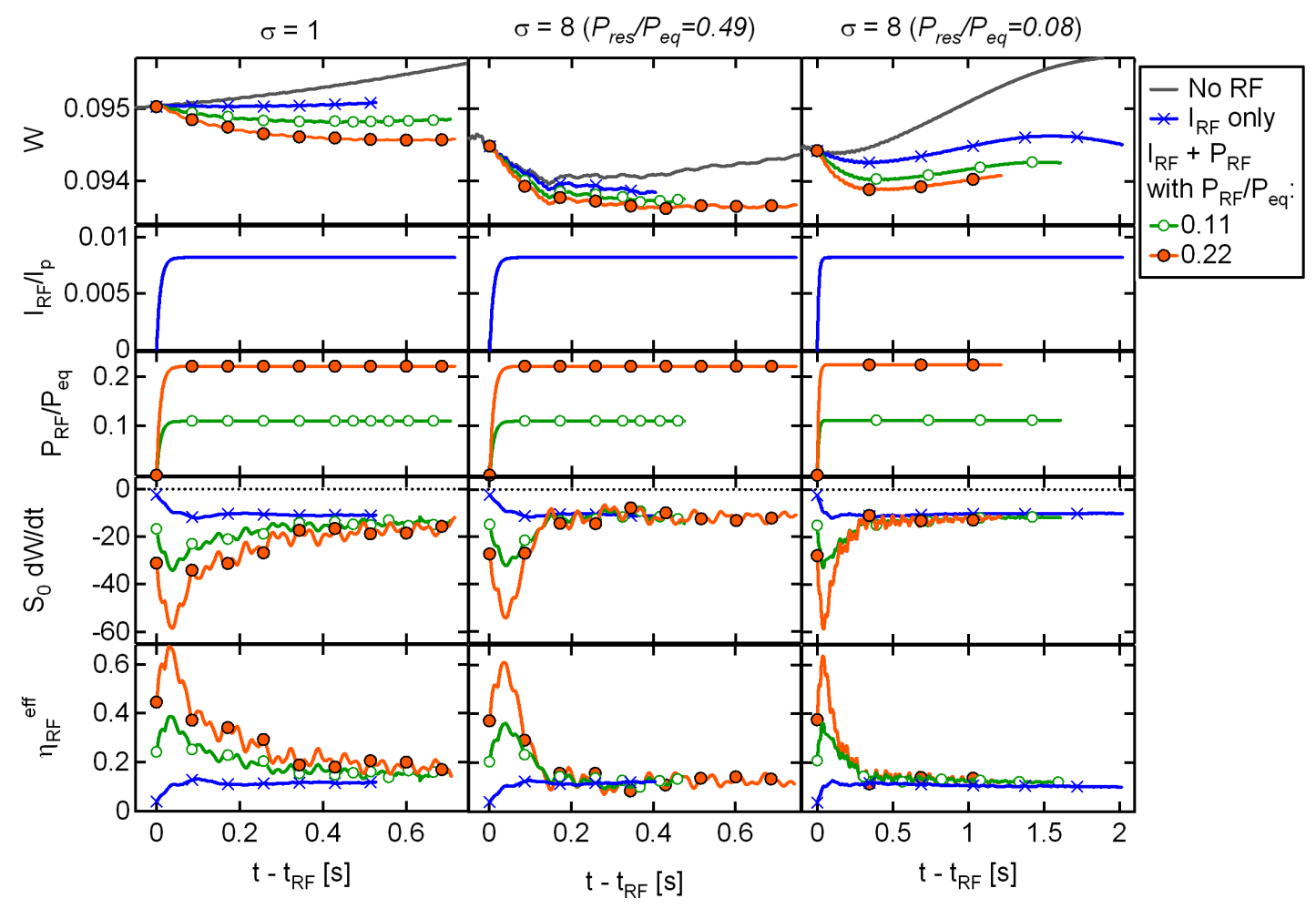

Figure 9. From top to bottom: island width, ratio $I_{R F} / I_{p}$, ratio $P_{R F} / P_{e q}$, normalized decay rate $S_{0} d \tilde{W} / d t$ and effective stabilization efficiency $\eta_{R F}^{\text {eff }}$ for $\sigma=1$ (left column), $\sigma=8$ with a residual power inside the island of $P_{r e s} / P_{e q}=0.49$ (middle column) and $\sigma=8$ with a residual power inside the island of $P_{r e s} / P_{e q}=0.08$ (right column).

5.1.2. Decay rate: contribution of heating We compute the decay rate and the stabilization efficiency by averaging on a time window covering the stationary phase. For $\sigma=1$, the transition between the initial overshoot and the long term decay rate is long, and it is still decreasing slowly at the end of the simulation. For this stiffness value, we choose the time window $\left(t-t_{R F}\right) \in[0.6,0.7] s$ whenever RF heating is applied, and $\left(t-t_{R F}\right) \in[0.4,0.5] s$ for the pure RF current stabilization scheme. For $\sigma=8$ on the contrary, the stationary regime is reached very rapidly, after about $200 \mathrm{~ms}$. The time window $\left(t-t_{R F}\right) \in[0.4,0.5] s$ is therefore appropriate. The decay rate and the effective stabilization efficiency are shown in figure 10 as a function of the RF power.

A comparison of the heating contribution to theory can be made by taking into account that the current and heating have nearly independent contributions, and that the decay rate of the combined effects is the sum of the two decay rates. This is due to the fact that, to leading order, the heating contribution to the perturbed bootstrap cancels in average, the temperature gradient being more positive on the inner side of the O-point and more negative on the outer part of it. We have investigated this assumption in dedicated simulations, and checked that this additivity is verified. The simulation results can then be compared with theory, as described in section 2, with an offset 


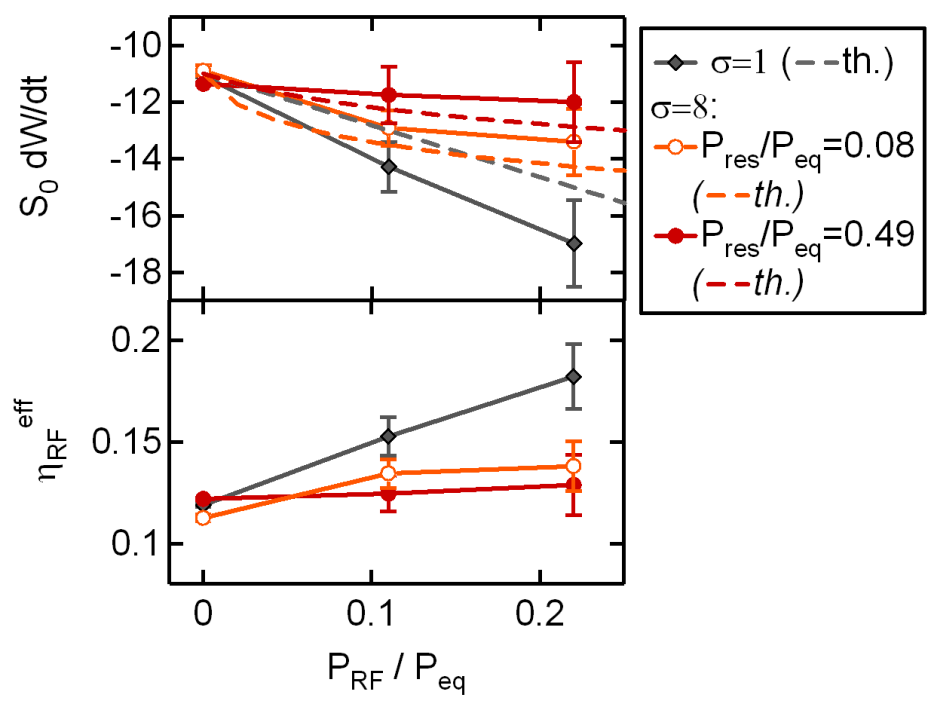

Figure 10. Normalized decay rate (top) and stabilization effective (bottom) averaged over the steady phase, as a function of the applied ECRH power for $\sigma=1$ and 8 , default $\left(P_{r e s} / P_{e q}=0.49\right)$ and ITER-like $\left(P_{r e s} / P_{e q}=0.08\right)$ heat source.

corresponding to the pure ECCD decay rate. The analytical prediction is

$$
\begin{aligned}
& \sigma=1: S_{0} \frac{d W}{d t}=-18.25 \frac{P_{R F}}{P_{e q}}+\left(S_{0} \frac{d W}{d t}\right)^{C D \text { only }} \\
& \sigma=8: S_{0} \frac{d W}{d t}=-11.50\left[\left(\frac{P_{R F}+\alpha_{1} P_{r e s}}{P_{e q}}\right)^{1 / \sigma}-\left(\frac{\alpha_{1} P_{r e s}}{P_{e q}}\right)^{1 / \sigma}\right]+\left(S_{0} \frac{d W}{d t}\right)^{C D \text { only }}
\end{aligned}
$$

where $\left(S_{0} d W / d t\right)^{C D \text { only }} \approx-11.00$.

For $\sigma=1$, the dependence of the decay rate with respect to the RF power is linear, in agreement with theory. The amplitude is overestimated, but as said before, this can be attributed to the fact that the initial overshoot is not fully relaxed. In the more realistic case of stiff transport $(\sigma=8)$, a good agreement between simulation results and theory is obtained once the residual heat is included in the theory. When the residual heat is large, there is no benefit from the heating contribution. For a situation representative of the ITER case $\left(P_{R F} / P_{e q}=15 \%, P_{r e s} / P_{e q}=8 \%\right)$, RF heating increases the decay rate and stabilization efficiency by about $30 \%$. This contribution is relatively large compared with the numbers found in the analytical approach because the pure RF current contribution is degraded. The net stabilization efficiency for ITER for continuous RF application is then $\eta_{R F}^{e f f, C W} \approx 0.14$, that is to say about half the expected value. 


\subsection{Modulated ECCD-ECRH Injection}

The use of a modulated RF source is motivated by the better stabilization efficiency obtained at the O-point as compared with the efficiency average over the full island phase. In principle, the benefit is low when the RF source is very narrow compared with the island size, and large when the RF deposition approaches or overcomes the island size. In our case, we have $\delta_{R F} / W \approx 0.23$, so that we would expect the benefit from modulation to be moderate. More precisely, using the formulas given in eq. 15, we expect the averaged stabilization efficiency to increase from $\eta_{R F}^{C W} \approx 0.33$ to $\eta_{R F}^{\text {mod }} \approx 0.40$ for a $50 \%$ duty cycle modulation. During the RF application, the stabilization efficiency should be large due to the low value of $\delta_{R F} / W$, but not as large as if RF was strictly focused on the O-point. In fact, the effective width of the RF deposition can be derived from the fact that it is related to the modulation duty cycle [Maget et al., 2018a]: $f^{o n}=\pi^{-1} \arccos \left(1-2 \mu_{c}^{e f f, m o d}\right)$ with $f^{o n}=0.5$ and $\mu_{c}^{e f f, m o d} \equiv\left(\delta_{R F}^{e f f, m o d} / W\right)^{2}$. This gives $\delta_{R F}^{e f f, \text { mod }} / W \approx 0.7$, and using for example the formula [Février et al., 2016] :

$$
\eta_{R F}^{O}=\frac{1}{1+\left(\delta_{R F} / W\right)^{2}}
$$

we expect the stabilization efficiency to be $\eta_{R F}^{O} \approx 0.67$.

To investigate this issue, we use pure ECCD or coupled ECCD-ECRH modulation with $50 \%$ duty cycle at the island O-point using a 3D Gaussian localized RF source, with a poloidal and toroidal widths of $\sigma_{\theta}=0.12$ and $\sigma_{\phi}=0.18$. The modulation process is artificially controlled by rotating toroidally the 3D RF source around the plasma at a low frequency $f_{\text {mod }}=10 \mathrm{~Hz}$, so that the stabilization efficiency when the RF source is activated can be compared with the pure O-point localization case represented by equation (40).

The simulations are performed for the most realistic case, i.e. $\sigma=8$ and $P_{r e s} / P_{e q}=0.08$, and the results are summarized in figure 11 , with an $\mathrm{RF}$ heating extending up to $P_{R F} / P_{e q}=0.78$. We show the evolution of the island width, the RF current, the RF power, the instantaneous stabilization efficiency $\eta_{R F}^{\text {eff }}$ during the RF application (the time average is performed on a sliding time window of $2 \mathrm{~ms}$ only) and the averaged stabilization efficiency $\left\langle\eta_{R F}^{e f f}\right\rangle$. We also compute the stabilization efficiency averaged during RF application, $\left\langle\eta_{R F}^{o n}\right\rangle$, defined as the time windows where the RF current is above $80 \%$ of its maximum value. Error bars represent the standard deviation of this average during each modulation.

The island response to pure CD application has a different behavior compared with combined $\mathrm{CD}$ and heating, since the island decay is slow to reach its stationary value, and it still has a remaining decay in between the RF application times. The stabilization efficiency at the end of the on-time is $\left\langle\eta_{R F}^{o n}\right\rangle \approx 0.37$. This is less than the expected value $\eta_{R F}^{O} \approx 0.67$ derived above, but still more than a factor of 3 above the continuous application value of about 0.11 . When heating is added, we benefit from the very efficient effect on short time scale, and the averaged stabilization efficiency during RF application rises to very large values, close to unity for $P_{R F} / P_{e q}=0.2$. The 


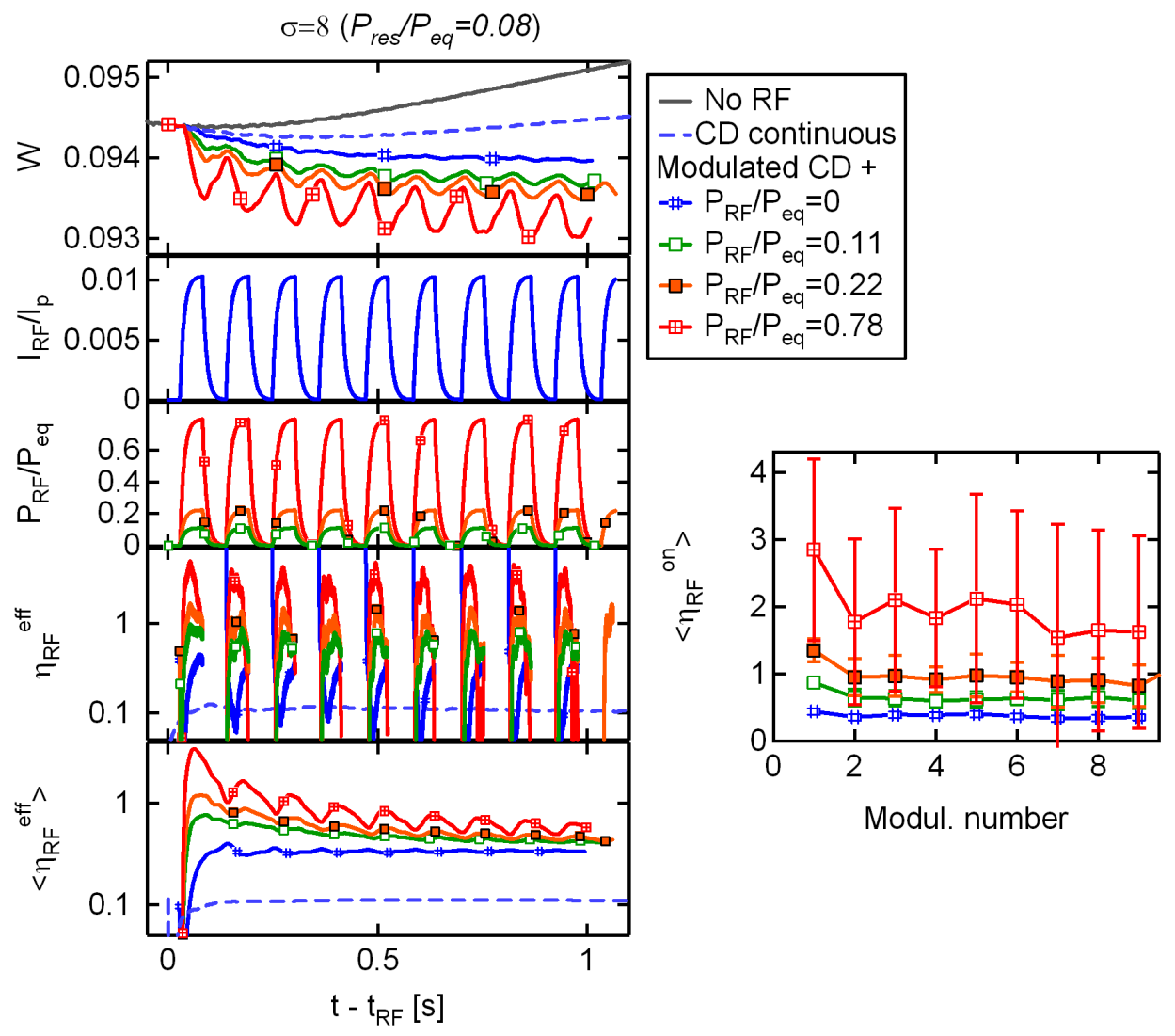

Figure 11. Left plot from top to bottom : island size, RF current, RF power, effective stabilization efficiency and averaged stabilization efficiency for $\sigma=8$ and $P_{r e s} / P_{e q}=0.08$. Right plot: stabilization efficiency during the $\mathrm{RF}$ on time as a function of the modulation cycle number. The value is taken at the end of the on-time for pure RF current, and averaged over the time where the RF current is above $80 \%$ of its maximum value for the combined current and heating cases.

low modulation frequency favors this large effect by allowing the level of turbulence inside the island to decay in between the RF application time windows. The large overshoot that characterizes the initial island decay after the RF power application can therefore be repeated at each modulation, with an amplitude that is only marginally reduced after 9 modulations. Note that the fact that the island goes below its averaged size during the modulation could in principle lower the requirements on the RF system [Ayten and Westerhof, 2012], but the amplitude of this island width modulation is too small to have a large benefit in the present case. However, the island grows again in between the modulations and corrects this favorable behavior. This is another strong difference with the RF current effect, that persists throughout the cycle.

The computation of the averaged effective stabilization efficiency $\left\langle\eta_{R F}^{e f f}\right\rangle$ provides an answer on the net advantage of the modulated control scheme. It shows that the pure CD application is much more efficient than in the continuous case. Whereas it 


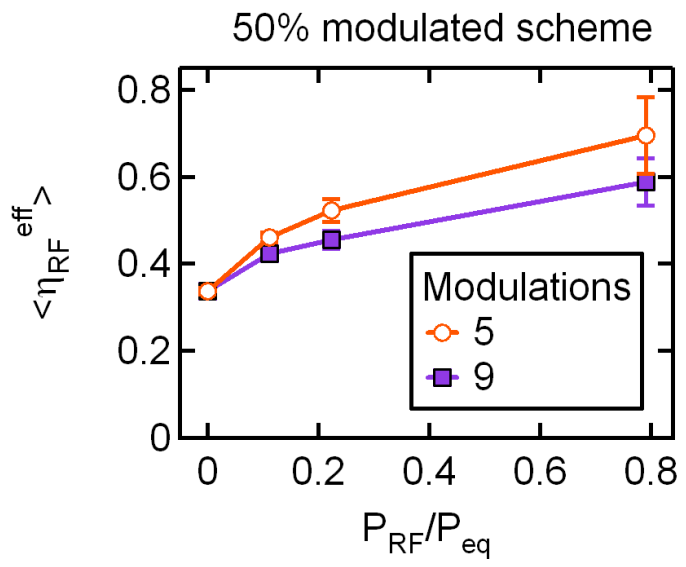

Figure 12. Averaged stabilization efficiency for $\sigma=8$ and $P_{r e s} / P_{e q}=0.08$ after 5 and 9 modulations at $10 \mathrm{~Hz}$, as a function of the RF power.

was largely degraded in the continuous case, with $\left\langle\eta_{R F}^{e f f, C W}\right\rangle \approx 0.11$, we recover in the modulated case a value consistent with theory, with $\left\langle\eta_{R F}^{\text {eff,mod }}\right\rangle \approx 0.34$. This is summarized in figure 12, showing the averaged stabilization efficiency for $\sigma=8$ and $P_{\text {res }} / P_{\text {eq }}=0.08$ after 5 and 9 modulations at $10 \mathrm{~Hz}$.

\section{Discussion on ECCD stabilization efficiency}

The effective ECCD stabilization efficiency that is obtained numerically $\left(P_{R F}=0\right)$ is lower than the theoretical prediction by about a third. Such a degradation was already reported, although less pronounced, in a previous work [Février et al., 2016] where simulations were performed with a pure resistive MHD model and pure O-point $\mathrm{RF}$ application. In order to document this phenomenon, we have made investigations in several directions, on the basis of a simpler, circular, equilibrium. The first check concerns the Fourier resolution, and we have performed simulations with a reduced toroidal discretization by filtering out Fourier modes above the first harmonic of the main mode, without any change of the stabilization efficiency. The second direction is the parallel diffusivity in the RF evolution that we have increased by a factor of two, without any change either on the stabilization efficiency. Another direction is the physics model. On the ITER-like equilibrium considered here, we find indeed that diamagnetic rotations degrade the stabilization efficiency, as shown in figure 13. The effective stabilization efficiency without diamagnetic rotations is increased by about $30 \%$ (from $\left\langle\eta_{R F}^{e f f}\right\rangle=0.11$ to $\left\langle\eta_{R F}^{e f f}\right\rangle=0.15$ ) but remains about a factor of 2 below the theoretical value $\left(\left\langle\eta_{R F}^{C W}\right\rangle=0.33\right)$. From the results reported in the present paper, a key point seems to be the contribution of the RF current deposited at the X-point. The fact that we recover the correct stabilization efficiency in the modulated scheme suggests indeed that the negative impact of the RF current in the X-point region of the island is 


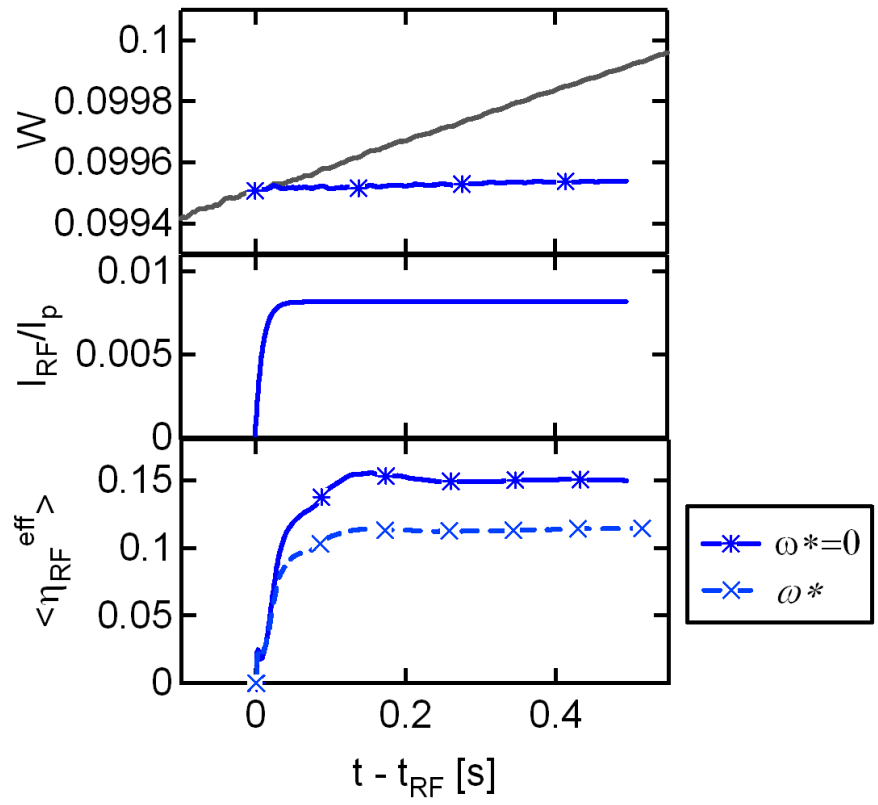

Figure 13. Island width (top), RF current (middle) and stabilization efficiency (bottom) for $\sigma=1$ with and without diamagnetic rotations and $I_{R F} / I_{p}=0.8 \%$.

much larger than expected.

\section{Conclusions}

We investigated in this work the stabilization of Neoclassical Tearing Mode by the combination of RF heating and current drive, with emphasis on the ITER specifications for the $(3,2)$ island. A criterion based on the Rutherford equation is derived to assess the necessary current and heating that are required to fully suppress a Neoclassical island. It confirms earlier results that the contribution of heating will be minor in ITER, and quantify this theoretical value in the context of a turbulent-based heat transport model that strongly impacts the island response to RF heating.

Numerical experiments performed with the XTOR code are then used to address the issues of island saturation and its stabilization by continuous or modulated RF application, taking into account both the current and heat contributions. They show that the $(3,2)$ island will saturate at about $10 \%$ of the minor radius, and that the residual heating coming from fusion reactions and additional heating systems reduce the saturation size. The control capability of the RF system is evaluated by computing the stabilization efficiency, a parameter that is instrumental for determining the RF system requirements from the Rutherford equation, and can then be compared with theoretical predictions. We find that for continuous RF application, the stabilization 
efficiency is about a factor of 3 lower than expected, and that this disagreement can be attributed to two main effects. The first one is related with diamagnetic rotations, and the second one comes from the underestimated contribution of the RF current deposited at the $\mathrm{X}$-point of the island. Indeed, the theoretical value is recovered in simulations where the RF current is modulated at the O-point. The contribution of RF heating is characterized by a large initial effect on the decay rate, but on a longer time scale, this effect is moderate but consistent with theory. As a result for continuous application, if the degradation of the stabilization efficiency by the RF current was to be confirmed, the margin of a factor of two on the required RF power that is predicted from the Rutherford analysis would become close to zero due the degradation of the stabilization efficiency by a similar factor. The use of a modulated control scheme enhances the role of RF heating during the RF application, since the strong initial effect is repeated at each modulation, allowing a lower island size to be reached transiently. The net stabilization efficiency that is found in this modulated scheme is consistent with theoretical values and gives confidence that the RF system foreseen on ITER will be able to stabilize the $(3,2)$ NTM if necessary.

\section{Acknowledgments}

This work has been carried out within the framework of the French Research Federation for Fusion Studies, and of the EUROfusion Consortium. It has received funding from the Euratom research and training programme 2014-2018 and 2019-2020 under grant agreement No 633053 for the project WP17-ENR-CEA-06. We benefited from HPC resources from GENCI (project 056348), from Marconi-Fusion (project NISMO) and from Aix-Marseille Université project Equip@Meso (ANR-10-EQPX-29-01) of the program "Investissements d'Avenir" supervised by the Agence Nationale pour la Recherche. This work is part of the project AMICI funded by the Agence Nationale pour la Recherche (ANR-14-CE32-0004-01). The views and opinions expressed herein do not necessarily reflect those of the European Commission.

\section{References}

[Agullo et al., 2017a] Agullo, O., Muraglia, M., Benkadda, S., Poy, A., Dubuit, N., Garbet, X., and Sen, A. (2017a). Nonlinear dynamics of turbulence driven magnetic islands. I. Theoretical aspects. Physics of Plasmas, 24(4):042308, DOI: 10.1063/1.4981229, https://doi.org/10. 1063/1.4981229.

[Agullo et al., 2017b] Agullo, O., Muraglia, M., Benkadda, S., Poy, A., Dubuit, N., Garbet, X., and Sen, A. (2017b). Nonlinear dynamics of turbulence driven magnetic islands. II. Numerical simulations. Physics of Plasmas, 24(4):042309, DOI: 10.1063/1.4981230, https://doi.org/10. 1063/1.4981230.

[Ayten and Westerhof, 2012] Ayten, B. and Westerhof, E. (2012). Consequences of plasma rotation for neoclassical tearing mode suppression by electron cyclotron current drive. Physics of Plasmas, 19(9):092506, 092506, DOI: 10.1063/1.4751873, http://link.aip.org/link/?PHP/19/092506/ 1. 
[Bardòczi et al., 2017] Bardòczi, L., Carter, T. A., Haye, R. J. L., Rhodes, T. L., and McKee, G. R. (2017). Impact of neoclassical tearing modeturbulence multi-scale interaction in global confinement degradation and magnetic island stability. Physics of Plasmas, 24(12):122503, DOI: 10.1063/1.5004987, https://doi.org/10.1063/1.5004987.

[Bardòczi et al., 2016] Bardòczi, L., Rhodes, T. L., Carter, T. A., Bañòn Navarro, A., Peebles, W. A., Jenko, F., and McKee, G. (2016). Modulation of core turbulent density fluctuations by largescale neoclassical tearing mode islands in the diii-d tokamak. Phys. Rev. Lett., 116:215001, DOI: 10.1103/PhysRevLett.116.215001, https://link.aps.org/doi/10.1103/PhysRevLett. 116.215001.

[Carrera et al., 1986] Carrera, R., Hazeltine, R. D., and Kotschenreuther, M. (1986). Island bootstrap current modification of the nonlinear dynamics of the tearing mode. Physics of Fluids, 29(4):899902, DOI: 10.1063/1.865682, http://link.aip.org/link/?PFL/29/899/1.

[Casper et al., 2014] Casper, T., Gribov, Y., Kavin, A., Lukash, V., Khayrutdinov, R., Fujieda, H., Kessel, C., Organization, I., and Agencies, I. D. (2014). Development of the ITER baseline inductive scenario. Nuclear Fusion, 54(1):013005, http://stacks.iop.org/0029-5515/54/i=1/ $\mathrm{a}=013005$.

[De Luca et al., 1986] De Luca, F., Jacchia, A., and Lazzaro, E. (1986). Control of tearing mode instabilities by localized electron cyclotron wave heating. Physics of Fluids (1958-1988), 29(2):501506, DOI: http://dx.doi.org/10.1063/1.865436, http://scitation.aip.org/content/aip/ journal/pof 1/29/2/10.1063/1.865436.

[Decker, J. et al., 2012] Decker, J., Peysson, Y., and Coda, S. (2012). Effect of density fluctuations on ECCD in ITER and TCV. EPJ Web of Conferences, 32:01016, DOI: 10.1051/epjconf/20123201016, http://dx.doi.org/10.1051/epjconf/20123201016.

[Farina et al., 2014] Farina, D., Henderson, M., Figini, L., and Saibene, G. (2014). Optimization of the ITER electron cyclotron equatorial launcher for improved heating and current drive functional capabilities. Physics of Plasmas (1994-present), 21(6):-, 061504, DOI: http://dx.doi.org/10.1063/1.4884352, http://scitation.aip.org/content/aip/journal/ pop/21/6/10.1063/1.4884352.

[Février et al., 2017] Février, O., Maget, P., Lütjens, H., and Beyer, P. (2017). Comparison of magnetic island stabilization strategies from magneto-hydrodynamic simulations. Plasma Physics and Controlled Fusion, 59(4):044002, http://stacks.iop.org/0741-3335/59/i=4/a=044002.

[Février et al., 2016] Février, O., Maget, P., Lütjens, H., Luciani, J. F., Decker, J., Giruzzi, G., Reich, M., Beyer, P., Lazzaro, E., Nowak, S., and the ASDEX Upgrade team (2016). First principles fluid modelling of magnetic island stabilization by electron cyclotron current drive (ECCD). Plasma Physics and Controlled Fusion, 58(4):045015, http://stacks.iop.org/0741-3335/58/i=4/a= 045015.

[Février et al., 2018] Février, O., Nicolas, T., Maget, P., Ahn, J.-H., Garbet, X., and Lütjens, H. (2018). Non-linear MHD simulations of sawteeth and their control by current and power depositions. Nuclear Fusion, 58(9):096008, http://stacks .iop.org/0029-5515/58/i=9/a=096008.

[Figini et al., 2015] Figini, L., Farina, D., Henderson, M., Mariani, A., Poli, E., and Saibene, G. (2015). Assessment of the ITER electron cyclotron upper launcher capabilities in view of an optimized design. Plasma Physics and Controlled Fusion, 57(5):054015, http://stacks.iop. org $/ 0741-3335 / 57 / i=5 / a=054015$.

[Fitzpatrick, 1995] Fitzpatrick, R. (1995). Helical temperature perturbations associated with tearing modes in tokamak plasmas. Physics of Plasmas, 2(3):825-838, DOI: 10.1063/1.871434, http: //link.aip.org/link/?PHP/2/825/1.

[Fitzpatrick, 2017] Fitzpatrick, R. (2017). Effect of nonlinear energy transport on neoclassical tearing mode stability in tokamak plasmas. Physics of Plasmas, 24(5):052504, DOI: 10.1063/1.4982610, http://dx.doi.org/10.1063/1.4982610.

[Furth et al., 1963] Furth, H. P., Killen, J., and Rosenbluth, M. (1963). Finite-resistivity instabilities of a sheet pinch. Physics of Fluids, 6(4):459-484. 
[Glasser et al., 1977] Glasser, A. H., Furth, H. P., and Rutherford, P. H. (1977). Stabilization of resistive kink modes in the tokamak. Phys. Rev. Lett., 38:234-237, DOI: 10.1103/PhysRevLett.38.234, http://link.aps.org/doi/10.1103/PhysRevLett.38.234.

[Hegna and Callen, 1997] Hegna, C. C. and Callen, J. D. (1997). On the stabilization of neoclassical magnetohydrodynamic tearing modes using localized current drive or heating. Physics of Plasmas, 4(8):2940-2946, DOI: 10.1063/1.872426, http://link.aip.org/link/?PHP/4/2940/1.

[Henderson et al., 2015] Henderson, M., Saibene, G., Darbos, C., Farina, D., Figini, L., Gagliardi, M., Gandini, F., Gassmann, T., Hanson, G., Loarte, A., Omori, T., Poli, E., Purohit, D., and Takahashi, K. (2015). The targeted heating and current drive applications for the ITER electron cyclotron system. Physics of Plasmas (1994-present), 22(2):-, 021808, DOI: http://dx.doi.org/10.1063/1.4908598, http://scitation.aip.org/content/aip/journal/ pop/22/2/10.1063/1.4908598.

[Hill et al., 2015] Hill, P., Hariri, F., and Ottaviani, M. (2015). The effect of magnetic islands on ion temperature gradient turbulence driven transport. Physics of Plasmas, 22(4):042308, DOI: 10.1063/1.4919031, https://doi.org/10.1063/1.4919031.

[Hornsby et al., 2010] Hornsby, W. A., Peeters, A. G., Snodin, A. P., Casson, F. J., Camenen, Y., Szepesi, G., Siccinio, M., and Poli, E. (2010). The nonlinear coupling between gyroradius scale turbulence and mesoscale magnetic islands in fusion plasmas. Physics of Plasmas, 17(9):092301, DOI: $10.1063 / 1.3467502$, http://dx.doi.org/10.1063/1.3467502.

[Hornsby et al., 2011] Hornsby, W. A., Siccinio, M., Peeters, A. G., Poli, E., Snodin, A. P., Casson, F. J., Camenen, Y., and Szepesi, G. (2011). Interaction of turbulence with magnetic islands: effect on bootstrap current. Plasma Physics and Controlled Fusion, 53(5):054008, http://stacks .iop. org $/ 0741-3335 / 53 / i=5 / a=054008$.

[Ida et al., 2012] Ida, K., Kamiya, K., Isayama, A., and Sakamoto, Y. (2012). Reduction of Ion Thermal Diffusivity Inside a Magnetic Island in JT-60U Tokamak Plasma. Phys. Rev. Lett., 109:065001, DOI: 10.1103/PhysRevLett.109.065001, https://link.aps.org/doi/10.1103/ PhysRevLett.109.065001.

[Inagaki et al., 2004] Inagaki, S., Tamura, N., Ida, K., Nagayama, Y., Kawahata, K., Sudo, S., Morisaki, T., Tanaka, K., and Tokuzawa, T. (2004). Observation of Reduced Heat Transport inside the Magnetic Island O Point in the Large Helical Device. Phys. Rev. Lett., 92:055002, DOI: 10.1103/PhysRevLett.92.055002, https://link.aps.org/doi/10.1103/PhysRevLett. 92.055002.

[Izacard et al., 2016] Izacard, O., Holland, C., James, S. D., and Brennan, D. P. (2016). Dynamics of ion temperature gradient turbulence and transport with a static magnetic island. Physics of Plasmas, 23(2):022304, DOI: 10.1063/1.4941704, http://dx.doi.org/10.1063/1.4941704.

[Kim et al., 2016] Kim, K., Na, Y.-S., Kim, H.-S., Maraschek, M., Poli, E., Stober, J., Zohm, H., Felici, F., Sauter, O., Park, Y., Terzolo, L., team, A. U., and Team, T. (2016). Modeling of neoclassical tearing mode stabilization by electron cyclotron heating and current drive in tokamak plasmas. Current Applied Physics, 16(8):867 - 875, ISSN: 1567-1739, DOI: http://dx.doi.org/10.1016/j.cap.2016.04.015, http://www.sciencedirect.com/science/ article/pii/S1567173916300888.

[Kinsey et al., 2011] Kinsey, J., Staebler, G., Candy, J., Waltz, R., and Budny, R. (2011). ITER predictions using the GYRO verified and experimentally validated trapped gyro-Landau fluid transport model. Nuclear Fusion, 51(8):083001, http://stacks.iop.org/0029-5515/51/i=8/ $\mathrm{a}=083001$.

[Kislov and T-10 Team, 2001] Kislov, D. and T-10 Team (2001). Recent experiments with ECRH and ECCD in T-10. Nuclear Fusion, 41(10):1473, http://stacks.iop.org/0029-5515/41/i=10/a= 314.

[Kotschenreuther et al., 1985] Kotschenreuther, M., Hazeltine, R. D., and Morrison, P. J. (1985). Nonlinear dynamics of magnetic islands with curvature and pressure. Physics of Fluids, 28(1):294302, DOI: 10.1063/1.865200, http://link.aip.org/link/?PFL/28/294/1. 
[Kurita et al., 1994] Kurita, G., Tuda, T., Azumi, M., Takizuka, T., and Takeda, T. (1994). Effect of local heating on the $\mathrm{m}=2$ tearing mode in a tokamak. Nuclear Fusion, 34(11):1497, http: //stacks.iop.org/0029-5515/34/i=11/a=I08.

[Lazzari and Westerhof, 2009] Lazzari, D. D. and Westerhof, E. (2009). On the merits of heating and current drive for tearing mode stabilization. Nuclear Fusion, 49(7):075002, http://stacks .iop. org $/ 0029-5515 / 49 / i=7 / a=075002$.

[Lazzari and Westerhof, 2010] Lazzari, D. D. and Westerhof, E. (2010). On the merits of heating and current drive for tearing mode stabilization. Nuclear Fusion, 50(7):079801, http://stacks.iop. org $/ 0029-5515 / 50 / i=7 / a=079801$.

[Lütjens et al., 1996] Lütjens, H., Bondeson, A., and Sauter, O. (1996). The CHEASE code for toroidal MHD equilibria. Computer Physics Communications, 97(3):219260, http://www.sciencedirect.com/science/article/B6TJ5-3VSGD5T-19/2/ e429c1260ed10db93fde0078b7cee3e6.

[Lütjens and Luciani, 2010] Lütjens, H. and Luciani, J.-F. (2010). XTOR-2F: A fully implicit Newton-Krylov solver applied to nonlinear 3D extended MHD in tokamaks. Journal of Computational Physics, 229(21):8130 - 8143, ISSN: 0021-9991, DOI: DOI: $10.1016 / j \cdot j c p .2010 .07 .013$, http://www.sciencedirect.com/science/article/ B6WHY-50JPNF3-2/2/2787626de93153effa740cca124bcfe7.

[Lütjens et al., 2001] Lütjens, H., Luciani, J.-F., and Garbet, X. (2001). Curvature effects on the dynamics of tearing modes in tokamaks. Physics of Plasmas, 8(10):4267-4270, http://link. aip.org/link/?PHP/8/4267/1.

[Maget et al., 2016] Maget, P., Février, O., Garbet, X., Lütjens, H., Luciani, J.-F., and Marx, A. (2016). Extended magneto-hydro-dynamic model for neoclassical tearing mode computations. Nuclear Fusion, 56(8):086004, http://stacks .iop.org/0029-5515/56/i=8/a=086004.

[Maget et al., 2018a] Maget, P., Widmer, F., Février, O., Garbet, X., and Lütjens, H. (2018a). Stabilization of a magnetic island by localized heating in a tokamak with stiff temperature profile. Physics of Plasmas, 25(2):022514, DOI: 10.1063/1.5021759, https://doi.org/10. 1063/1.5021759.

[Maget et al., 2018b] Maget, P., Widmer, F., Février, O., Lütjens, H., and Garbet, X. (2018b). Numerical experiments of island stabilization by RF heating with stiff temperature profile. Plasma Physics and Controlled Fusion, 60(9):095003, http://stacks.iop.org/0741-3335/60/i=9/a= 095003.

[Maraschek, 2012] Maraschek, M. (2012). Control of neoclassical tearing modes. Nuclear Fusion, 52(7):074007, http://stacks.iop.org/0029-5515/52/i=7/a=074007.

[Navarro et al., 2017] Navarro, A. B., Bardòczi, L., Carter, T. A., Jenko, F., and Rhodes, T. L. (2017). Effect of magnetic islands on profiles, flows, turbulence and transport in nonlinear gyrokinetic simulations. Plasma Physics and Controlled Fusion, 59(3):034004, http://stacks.iop.org/ $0741-3335 / 59 / i=3 / a=034004$.

[Peysson et al., 2011] Peysson, Y., Decker, J., Morini, L., and Coda, S. (2011). RF current drive and plasma fluctuations. Plasma Physics and Controlled Fusion, 53(12):124028, http://stacks .iop. org $/ 0741-3335 / 53 / i=12 / a=124028$.

[Pletzer and Perkins, 1999] Pletzer, A. and Perkins, F. W. (1999). Stabilization of neoclassical tearing modes using a continuous localized current drive. Physics of Plasmas, 6(5):1589-1600, DOI: 10.1063/1.873412, http://link.aip.org/link/?PHP/6/1589/1.

[Poli et al., 2015] Poli, E., Angioni, C., Casson, F., Farina, D., Figini, L., Goodman, T., Maj, O., Sauter, O., Weber, H., Zohm, H., Saibene, G., and Henderson, M. (2015). On recent results in the modelling of neoclassical-tearing-mode stabilization via electron cyclotron current drive and their impact on the design of the upper EC launcher for ITER. Nuclear Fusion, 55(1):013023, http://stacks.iop.org/0029-5515/55/i=1/a=013023.

[Poli et al., 2018] Poli, F., Fredrickson, E., Henderson, M., Kim, S.-H., Bertelli, N., Poli, E., Farina, D., and Figini, L. (2018). Electron cyclotron power management for control of neoclassical tearing 
modes in the ITER baseline scenario. Nuclear Fusion, 58(1):016007, http://stacks.iop.org/ $0029-5515 / 58 / i=1 / a=016007$.

[Popov et al., 2002] Popov, A. M., Haye, R. J. L., Liu, Y. Q., Murakami, M., Popova, N. N., and Turnbull, A. D. (2002). Simulation of neoclassical tearing modes in the DIII-D tokamak. II. suppression by radially localized electron cyclotron current drive. Physics of Plasmas, 9(10):42294240, DOI: 10.1063/1.1505843, http://link.aip.org/link/?PHP/9/4229/1.

[Rutherford, 1973] Rutherford, P. H. (1973). Nonlinear growth of the tearing mode. Physics of Fluids, 16(11):1903-1908, DOI: 10.1063/1.1694232, http://link.aip.org/link/?PFL/16/1903/1.

[Sauter, 2004] Sauter, O. (2004). On the contribution of local current density to neoclassical tearing mode stabilization. Physics of Plasmas, 11(10):4808-4813, DOI: 10.1063/1.1787791, http: //link. aip.org/link/?PHP/11/4808/1.

[Sing et al., 1993] Sing, D. C., Austin, M. E., Brower, D. L., Chen, J. Y., Gandy, R. F., Yu, C. X., Wang, X., Bhattacharjee, A., and Schnack, D. D. (1993). "suppression of $\mathrm{m}=2$ islands by electron cyclotron heating in the texas experimental tokamak: Experiment and theory". Physics of Fluids B: Plasma Physics (1989-1993), 5(9):3239-3245, DOI: http://dx.doi.org/10.1063/1.860659, http://scitation.aip.org/content/aip/journal/pofb/5/9/10.1063/1.860659.

[Snicker et al., 2018] Snicker, A., Poli, E., Maj, O., Guidi, L., Köhn, A., Weber, H., Conway, G., Henderson, M., and Saibene, G. (2018). The effect of density fluctuations on electron cyclotron beam broadening and implications for ITER. Nuclear Fusion, 58(1):016002, http://stacks .iop. org $/ 0029-5515 / 58 / i=1 / a=016002$.

[Sysoeva et al., 2015] Sysoeva, E., da Silva, F., Gusakov, E., Heuraux, S., and Popov, A. (2015). Electron cyclotron resonance heating beam broadening in the edge turbulent plasma of fusion machines. Nuclear Fusion, 55(3):033016, http://stacks.iop.org/0029-5515/55/i=3/ $\mathrm{a}=033016$.

[Westerhof, 1987] Westerhof, E. (1987). Requirements on heating or current drive for tearing mode stabilization by current profile tailoring. Nuclear Fusion, 27(11):1929, http://stacks.iop.org/ $0029-5515 / 27 / i=11 / a=018$.

[Westerhof et al., 2007] Westerhof, E., Lazaros, A., Farshi, E., de Baar, M., de Bock, M., Classen, I., Jaspers, R., Hogeweij, G., Koslowski, H., Krmer-Flecken, A., Liang, Y., Cardozo, N. L., and Zimmermann, O. (2007). Tearing mode stabilization by electron cyclotron resonance heating demonstrated in the TEXTOR tokamak and the implication for ITER. Nuclear Fusion, 47(2):85, http://stacks.iop.org/0029-5515/47/i=2/a=003.

[Yu and Günter, 1998] Yu, Q. and Günter, S. (1998). On the stabilization of neoclassical tearing modes by phased electron cyclotron waves. Plasma Physics and Controlled Fusion, 40(11):1977, http://stacks.iop.org/0741-3335/40/i=11/a=011.

[Yu et al., 2000] Yu, Q., Gunter, S., Giruzzi, G., Lackner, K., and Zabiego, M. (2000). Modeling of the stabilization of neoclassical tearing modes by localized radio frequency current drive. Physics of Plasmas, 7(1):312-322, DOI: 10.1063/1.873799, http://link.aip.org/link/?PHP/7/312/1.

[Yu et al., 2004] Yu, Q., Zhang, X. D., and Günter, S. (2004). Numerical studies on the stabilization of neoclassical tearing modes by radio frequency current drive. Physics of Plasmas, 11(5):19601968, DOI: http://dx.doi.org/10.1063/1.1710521, http://scitation.aip.org/content/ aip/journal/pop/11/5/10.1063/1.1710521.

[Zarzoso et al., 2015] Zarzoso, D., Hornsby, W., Poli, E., Casson, F., Peeters, A., and Nasr, S. (2015). Impact of rotating magnetic islands on density profile flattening and turbulent transport. Nuclear Fusion, 55(11):113018, http://stacks .iop.org/0029-5515/55/i=11/a=113018. 